\title{
ON REDUCIBILITY AND UNITARIZABILITY \\ FOR CLASSICAL $p$-ADIC GROUPS, SOME GENERAL RESULTS
}

\author{
MARKo TADIĆ
}

\begin{abstract}
The aim of this paper is to prove two general results on parabolic induction of classical $p$-adic groups (actually, one of them holds also in the archimedean case), and to obtain from them some consequences about irreducible unitarizable representations. One of these consequences is a reduction of the unitarizability problem for these groups. This reduction is similar to the reduction of the unitarizability problem to the case of real infinitesimal character for real reductive groups.
\end{abstract}

\section{INTRODUCTION}

Let $F$ be a $p$-adic field. The normalized absolute value on $F$ will be denoted by ||$_{F}$. Denote by $\nu: G L(n, F) \rightarrow \mathbb{R}^{\times}$the character

$$
\nu(g)=|\operatorname{det}(g)|_{F} .
$$

For two smooth representations $\pi_{1}$ and $\pi_{2}$ of $G L\left(n_{1}, F\right)$ and $G L\left(n_{2}, F\right)$ respectively, we can consider $\pi_{1} \otimes \pi_{2}$ as a representation of Levi factor of the maximal parabolic subgroup which contains upper triangular matrices and whose Levi factor is isomorphic to $G L\left(n_{1}, F\right) \times$ $G L\left(n_{2}, F\right)$. Following Bernstein and Zelevinsky, we denote by

$$
\pi_{1} \times \pi_{2}
$$

the representation of $G L\left(n_{1}+n_{2}, F\right)$ parabolically induced by $\pi_{1} \otimes \pi_{2}$ (see the first section for more details regarding notation). For an irreducible essentially square integrable representation $\delta$ of $G L(n, F)$ there exists a unique $e(\delta) \in \mathbb{R}$ and a unique unitarizable (irreducible square integrable) representation $\delta^{u}$, such that

$$
\delta=\nu^{e(\delta)} \delta^{u} .
$$

1991 Mathematics Subject Classification. Primary 22E50, 22E35.

The author was partly supported by Croatian Ministry of Science, Education and Sports grant \# 037 0372794-2804.

Typeset by $\mathcal{A}_{\mathcal{M}} \mathcal{S}-\mathrm{T}_{\mathrm{E}} \mathrm{X}$ 
We shall fix a tower of symplectic or odd-orthogonal spaces over $F$. We shall denote by $S_{n}$ the group with split rank $n$, of isometries of determinant one of a space from the tower. Since Levi factors of parabolic subgroups are direct products of general linear groups and smaller groups $S_{m}$, for a smooth representations $\pi$ and $\sigma$ of $G L(p, F)$ and $S_{q}$ respectively, we can parabolically induce $\pi \otimes \sigma$ and get a representation of $S_{p+q}$ (similarly as in the case of general linear groups). We shall denote the parabolically induced representation by

$$
\pi \rtimes \sigma .
$$

The aim of this paper is to prove two general results regarding parabolic induction of classical $p$-adic groups (actually, one of them holds also in the archimedean case), and to obtain from them some consequences about irreducible unitarizable representations, like a reduction of the problem of unitarizability for these groups.

The first of the two main results of the paper gives for irreducible representations $\pi$ and $\sigma$ a sufficient conditions that $\pi \rtimes \sigma$ is irreducible (see Proportion 2.2). This result could be also obtained by (powerful) methods of $[\mathrm{J}]$, after necessary modifications. Instead of explaining here this technical result, we shall recall of the most important consequence. An irreducible representation $\sigma$ of $S_{q}$ will be called weakly real if whenever we have embedding

$$
\sigma \hookrightarrow \rho_{1} \times \ldots \times \rho_{l} \rtimes \sigma^{\prime},
$$

where $\rho_{1}, \ldots, \rho_{l}$ are irreducible cuspidal representations of general linear groups and $\sigma^{\prime}$ is an irreducible cuspidal representation of some $S_{q^{\prime}}$, then

$$
\rho_{i}^{u} \cong \tilde{\rho}_{i}^{u}
$$

for all $i \in\{1, \ldots, l\}$ ( $\tilde{\rho}$ denotes the contragredient representation of $\rho$ ). We have:

Theorem A. If $\sigma$ is an irreducible unitarizable representation of some $S_{q}$, then there exist an irreducible unitarizable representation $\pi$ of a general linear group and a weakly real irreducible unitarizable representation $\sigma^{\prime}$ of some $S_{q^{\prime}}$ such that

$$
\sigma \cong \pi \rtimes \sigma^{\prime}
$$

Since we have a classification of irreducible unitarizable representations of general linear groups ([T1]), the above theorem reduces the problem of classification of irreducible unitarizable representations of classical groups to the problem of classification of weakly real irreducible unitarizable representations of classical groups (such a type of reduction in [T8] for irreducible square integrable representations of classical $p$-adic groups, was an important initial step in the process of classifying of irreducible square integrable representations of classical $p$-adic groups, which ended with such a classification modulo cuspidal data in [Mœ] and [MøT]).

Suppose that an irreducible representation $\sigma$ of $S_{m}$ is a subquotient of a principal series representation $\operatorname{Ind}_{P_{\emptyset}}^{S_{m}}(\chi)$. If $\sigma$ is weakly real, then $\chi$ is real valued. In particular, if $\sigma$ is a weakly real irreducible representation with Iwahori fixed vector, then its infinitesimal character is determined by $\left(A_{\emptyset}, \chi\right)$, and $\chi$ is a real valued unramified character. Therefore, 
the above theorem is similar to the reduction of the unitarizability problem to the case of real infinitesimal character for real reductive groups. The above theorem is also related to the reduction obtained in [BrMo1].

In this paper we get also an upper bound for the places where weakly real irreducible unitarizable representations of classical $p$-adic groups can show up. We explain this bound now. Let $\rho$ be an irreducible self dual cuspidal representation of a general linear group and let $\sigma^{\prime}$ be an irreducible cuspidal representation of some $S_{q}$. Then there exists a unique $\alpha_{\rho, \sigma^{\prime}} \geq 0$ such that

$$
\nu^{\alpha_{\rho, \sigma^{\prime}} \rho \rtimes \sigma^{\prime}}
$$

reduces.

Proposition A. Suppose that $\sigma$ is a weakly real irreducible unitarizable representations of a classical group. Fix any embedding as in (1) and fix $i_{0} \in\{1, \ldots, l\}$. Denote by $\pi_{1}, \ldots, \pi_{n}$ all the representations from $\left\{\rho_{1}, \ldots, \rho_{l}\right\}$ for which $\rho_{i}^{u} \cong \rho_{i_{0}}^{u}$. Write

$$
\left\{\left|e\left(\pi_{i}\right)\right| ;\left|e\left(\pi_{i}\right)\right|>\alpha_{\rho_{i_{0}}^{u}, \sigma^{\prime}} \text { and } 1 \leq i \leq n\right\}=\left\{\alpha_{1}, \ldots, \alpha_{\ell}\right\},
$$

where $\ell \geq 0$ and $\alpha_{1}<a_{2}<\cdots<\alpha_{\ell}$. Then

(i) $\alpha_{1}-\alpha_{\rho_{i_{0}}^{u}, \sigma^{\prime}} \leq 1$ if $\ell \geq 1$, and

$$
\alpha_{i}-\alpha_{i-1} \leq 1 \quad \text { for each } i=2,3, \ldots, \ell .
$$

(ii)

$$
\alpha_{i} \leq \alpha_{\rho_{0}}^{u}, \sigma^{\prime}+i ; \quad i=1, \ldots, \ell .
$$

One of the main results of our paper is identification of some irreducible subquotients of parabolically induced representations. Such a result is natural to expect if we consider (conjectural) local Langlands correspondences (and functoriality in its simples form).

Let $D$ be the set of all equivalence classes of irreducible essentially square integrable representation of $G L(n, F)$ 's, $n \geq 1$. Denote $D_{+}=\{\delta \in D ; e(\delta)>0\}$. The set of finite multisets in $D$ (resp. $D_{+}$) will be denoted by $M(D)$ (resp. $M\left(D_{+}\right)$). We add multisets in a natural way. The Langlands classification for general linear groups attaches to each $d \in M(D)$ an irreducible representation $L(d)$ (see the fourth section for details).

Denote by $T(S)$ the set of all equivalence classes of irreducible tempered representations of groups $S_{q}, q \geq 0$. Then the Langlands classification for groups $S_{q}$ parametrize irreducible representations of these groups by elements of $M\left(D_{+}\right) \times T(S)$. To $\left(d^{\prime}, \tau\right) \in M\left(D_{+}\right) \times T(S)$ is attached $L\left(d^{\prime}, \tau\right)$ (see the fourth section for details).

For $d=\left(\delta_{1}, \ldots, \delta_{k}\right) \in M(D)$ denote

$$
\lambda(d)=\delta_{1} \times \ldots \times \delta_{k} .
$$

We shall denote by

$$
d^{\uparrow}
$$

the element of $M\left(D_{+}\right)$which we get from $d$ by removing all unitarizable $\delta_{i}$ 's, and changing all $\delta_{i}$ 's for which $e\left(\delta_{i}\right)<0$, by $\tilde{\delta}_{i}$. Denote by

$$
d_{u}
$$

the multiset in $M(D)$ which we get from $d$ removing all $\delta_{i}$ 's which are not unitarizable. 
Proposition B. Let $d \in M(D)$ and $t=\left(d^{\prime}, \tau\right) \in M\left(D_{+}\right) \times T(S)$. Denote by

$$
T_{d, \tau}
$$

the set of all (equivalence classes of) irreducible subrepresentations of $\lambda\left(d_{u}\right) \rtimes \tau$. Then each of the representations

$$
L\left(d^{\uparrow}+d^{\prime} ; \tau^{\prime}\right), \quad \tau^{\prime} \in T_{d, \tau}
$$

is a subquotient of

$$
L(d) \rtimes L\left(d^{\prime} ; \tau\right) .
$$

If $F$ is of characteristic 0 , then the multiplicity of each of these representations in $L(d) \rtimes$ $L\left(d^{\prime} ; \tau\right)$ is one.

From this result we can conclude reducibility of some representation which are interesting for construction of irreducible unitary representations of groups $S_{q}$ (see the fourth section). Further work on understanding such reducibilities will be related to understanding of Arthur's $R$-groups (see Corollary 4.4 and $[\mathrm{BnJ}]$ ).

The above result is a generalization of the following result of A.V. Zelevinsky from the case of general linear groups (which played an important role in the process of classifying of irreducible unitary representations of these groups):

$$
L\left(d_{1}+d_{2}\right) \text { is a subquotient of } L\left(d_{1}\right) \times L\left(d_{2}\right) \text { with multiplicity } 1 \text {. }
$$

Let us recall that the above result holds also in the archimedean case (see [T2]).

Let $F \subset F^{\prime}$ be a separable quadratic extension, and let $\theta$ be a non-trivial element of the Galois group. We can consider towers of unitary groups associated with this extension (see $[\mathrm{M} T]$ ). When we fix one such series, all the results of this paper, after changing contragredients $\tilde{\pi}$ to representations $g \mapsto \tilde{\pi}(\theta(g))$ in them, hold also for unitary groups with same proofs.

We expect that the results of the paper also hold for even orthogonal groups, with basically the same proofs.

The results of this paper may be considered as initial steps of the work on the unitarizability problem for classical $p$-adic groups in general case. Some of initial ideas in that direction are presented in the last section of [T9]. We are finishing writing of the sequel of this paper, in which we are giving evidence for the approach to the unitarizability which we are starting here (and in [T9]).

We give now more information about the content of this paper, section by section. The first section introduces notation. The second section presents the sufficient condition for irreducibility of parabolically induced representations. In the third section we list some consequences of this result, mainly related to the problem of unitarizability. The description of Langlands parameters of some distinguished subquotients of parabolically induced representations $L(d) \rtimes L\left(d^{\prime}, \tau\right)$ is proved in the fourth section. We give there some applications of this result to the unitarizability problem. In the fifth section we collect some simple already known results on reducibility of parabolic induction, which are useful in studying unitarizability.

We are thankful to G. Muić for discussions on the topics studied in this paper. These discussions have helped to clarify some parts of the paper. 


\section{Notation}

First we shall introduce basic notation of the representation theory of general linear groups over a $p$-adic field $F$. We shall use mainly notation of Bernstein and Zelevinsky from $[\mathrm{Z}]$. The normalized absolute value on $F$ will be denoted by ||$_{F}$. The character $g \mapsto$ $|\operatorname{det}|_{F}, G L(n, F) \rightarrow \mathbb{R}^{\times}$is denoted by $\nu$. For each irreducible essentially square integrable representation $\delta$ of $G L(n, F)$ there exist a unique, up to an equivalence, (unitarizable) irreducible square integrable representation $\delta^{u}$, and $e(\delta) \in \mathbb{R}$, such that $\delta=\nu^{e(\delta)} \delta^{u}$.

There is a natural map from the category of smooth representations of a reductive $p$-adic group $G$ of finite length, to the Grothendieck group of this category. It will be denoted by s.s. (this map is called semi simplification). An irreducible representation $\pi$ of $G$ can be considered in a natural way as an element of the Grothendieck group.

Let $\pi_{i}$ be a smooth representation of $G L\left(n_{i}, F\right)$ for $i=1,2$. Then $\pi_{1} \times \pi_{2}$ will denote the smooth representation of $G L\left(n_{1}+n_{2}, F\right)$ parabolically induced by $\pi_{1} \otimes \pi_{2}$ from appropriate maximal parabolic subgroup, which is standard with respect to the subgroup of the upper triangular matrices (parabolic induction that we consider is normalized). The sum of Grothendieck groups $R_{n}$ of the category of smooth representations of $G L(n, F)$ of finite length is dented by $R$. Then $\times$ lifts in a natural way to a mapping $R \times R \rightarrow R$ which will be again denoted by $\times$. This mapping naturally factors through a mapping $R \otimes R \rightarrow R$, which will be denoted by $m$. For an admissible representation $\pi$ of $G L(n, F)$, the sum of semi simplifications of the Jacquet modules for standard parabolic subgroups which has Levi subgroups $G L(k, F) \times G L(n-k, F), 0 \leq k \leq n$, defines an element of $R \otimes R$ in a natural way (see $[\mathrm{Z}]$ for a precise definition; Jacquet modules that we consider in this paper will be always normalized). This can be additively extended to a mapping

$$
m^{*}: R \rightarrow R \otimes R
$$

In this way $R$ becomes a Hopf algebra.

Let $\pi$ be an irreducible representation of $G L(n, F)$. Then there exist irreducible cuspidal representations $\rho_{1}, \ldots, \rho_{k}$ of general linear groups such that $\pi$ is isomorphic to a subquotient of $\rho_{1} \times \cdots \times \rho_{k}$. The multiset of equivalence classes $\left(\rho_{1}, \ldots, \rho_{k}\right)$ is called the cuspidal support of $\pi$ (it depends only on the the equivalence class of $\pi$ ). It is denoted by $\operatorname{supp}(\pi)$

Now we shall introduce basic notation of the representation theory of the classical $p$-adic groups. We shall follow the notation of [T4] and [MœT]. Fix now a Witt tower $V \in \mathcal{V}$ of symplectic vector spaces over $F$, or of orthogonal vector spaces which starts with an unisotropic space of odd dimension. We shall denote by $S(V)$ the group of isometries of $V \in \mathcal{V}$ of determinant 1 (this is automatically satisfied in the symplectic case). The group of split rank $n$ will be denoted by $S_{n}$. Now the sum of Grothendieck groups $R_{n}(S)$ of categories of smooth representations of $S_{n}$ of finite length, is dented by $R(S)$. Similarly as in the case of general linear groups, using parabolic induction, one defines $\pi \rtimes \sigma$ for a smooth representation $\pi$ of a general linear group over $F$ and a smooth representation $\sigma$ of $S_{m}$. Now $\rtimes$ lifts in a natural way to a mapping $R \times R(S) \rightarrow R$ which is again denoted by $\rtimes$ (factorization through $R \otimes R(S)$ is dented by $\mu$ ). In this way $R(S)$ becomes $R$-module. Recall that

$$
\pi \rtimes \sigma=\tilde{\pi} \rtimes \sigma
$$


in $R(\tilde{\pi}$ denotes the contragredient of $\pi)$.

The Jacquet module of a representation $\pi$ of $S_{n}$ for the standard maximal parabolic subgroup whose Levi factor is a direct product of $G L(k, F)$ and a classical group, is denoted by

$$
s_{(k)}(\pi) .
$$

Suppose that $\pi$ has finite length. The sum of semi simplifications of $s_{(k)}(\pi), 0 \leq k \leq n$, is denoted by

$$
\mu^{*}(\pi)
$$

We can consider $\mu^{*}(\pi) \in R \otimes R(S)$. Extending additively to

$$
\mu^{*}: R(S) \rightarrow R \otimes R(S),
$$

one gets the structure of $R$-comodule on $R(S)$.

Observe that $R \otimes R(S)$ is in a natural way $R \otimes R$-module (the multiplication is denoted again by $\rtimes)$. Further, denote by $\sim: R \rightarrow R$ the contragredient map and by $\kappa: R \otimes R \rightarrow$ $R \otimes R, \sum x_{i} \otimes y_{i} \mapsto y_{i} \otimes x_{i}$. Let

$$
M^{*}=\left(m \otimes \operatorname{id}_{R}\right) \circ\left(\sim \otimes m^{*}\right) \circ \kappa \circ m^{*} .
$$

Then

$$
\mu^{*}(\pi \rtimes \sigma)=M^{*}(\pi) \rtimes \mu^{*}(\sigma)
$$

for admissible representations $\pi$ and $\sigma$ of $G L(n, F)$ and $S_{m}$ respectively (or, for elements of $R$ and $R(S)$ respectively). In other words, if $m^{*}(\pi)=\sum_{i} x_{i} \otimes y_{i}$, then

$$
M^{*}(\pi)=\sum_{i}\left(m \otimes \operatorname{id}_{R}\right)\left(\tilde{y}_{i} \times m^{*}\left(x_{i}\right)\right) .
$$

The term of $M^{*}(\pi)$ in $R \otimes R_{0}$ will be denoted by $M_{G L}^{*}(\pi)$. Then

$$
M_{G L}^{*}(\pi)=\left[m \circ\left(\operatorname{id}_{R} \otimes \sim\right) \circ m^{*}(\pi)\right] \otimes 1
$$

(since $R$ is commutative). Thus if $m^{*}(\pi)=\sum_{i} x_{i} \otimes y_{i}$, then

$$
M_{G L}^{*}(\pi)=\sum_{i} x_{i} \times \tilde{y}_{i}
$$

The term $M^{*}(\pi)$ in $R_{0} \otimes R$ is simply $1 \otimes \pi$.

Let $\tau$ be an irreducible representation of some classical group $S_{\ell}$. Then it is a subquotient of

$$
\rho_{1} \times \ldots \cdots \times \rho_{k} \rtimes \sigma
$$

for some irreducible cuspidal representations $\rho_{1}, \ldots, \rho_{k}$ of general linear groups and an irreducible cuspidal representation $\sigma$ of some $S_{m}$. The representation $\sigma$ is called the partial cuspidal support of $\tau$ and denoted by 
If $\rho_{1} \times \ldots \cdots \times \rho_{k}$ is a representation of $G L(p, F)$, then the Jacquet module $s_{(p)}(\tau)$ will be denoted by

$$
s_{G L}(\tau)
$$

An irreducible cuspidal representation $\rho$ of a general linear group is called a factor of $\tau$ if there exists an irreducible subquotient $\pi \otimes \tau_{c u s p}$ of $s_{G L}(\tau)$ such that $\rho$ is in the cuspidal support of $\pi$. Then the set of all factors of $\tau$ is contained in

$$
\left\{\rho_{1}, \tilde{\rho}_{1}, \rho_{2}, \tilde{\rho}_{2}, \ldots, \rho_{k}, \tilde{\rho}_{k}\right\}
$$

(recall that our $\tau$ is a subquotient of $\rho_{1} \times \ldots \cdots \times \rho_{k} \rtimes \sigma$ ). Further, for each $1 \leq i \leq k$, at least one representation from $\left\{\rho_{i}, \tilde{\rho}_{i}\right\}$ is a factor of $\tau$.

We have a natural ordering $\leq$ on the Grothendieck group of the category of smooth representations of a reductive $p$-adic group $G$, of finite length. Therefore, there are natural orderings on $R, R(S), R \otimes R$ and $R \otimes R(S)$. If we write $\pi_{1} \leq \pi_{2}$ for two admissible representations, we shall actually mean inequality between their images in the Grothendieck group (i.e. between their semi simplifications). We shall use quite often two forms of Frobenius reciprocity. Let $P=M N$ be a parabolic subgroup of $G$. Suppose that $\pi$ and $\sigma$ are smooth representations of $G$ and $M$ respectively. The Jacquet module of $\pi$ with respect to $P=M N$ will be denoted by $r_{P}^{G}(\pi)$. Then Frobenius reciprocity says that we have a canonical isomorphism

$$
\operatorname{Hom}_{G}\left(\pi, \operatorname{Ind}_{P}^{G}(\sigma)\right) \cong \operatorname{Hom}_{M}\left(r_{P}^{G}(\pi), \sigma\right)
$$

Let $A_{\emptyset}$ be a maximal split torus in a connected reductive group $G$ over $F$. Suppose that $P$ contains $A_{\emptyset}$. Then we can find Levi subgroup $M$ which contains $A_{\emptyset}$ (such $M$ is unique). Let $\bar{P}$ be the opposite parabolic subgroup (this is the unique parabolic subgroup which contains $A_{\emptyset}$, whose Levi subgroup is $M$ and which satisfies $P \cap \bar{P}=M$ ). Then the second form of Frobenius reciprocity is

$$
\operatorname{Hom}_{G}\left(\operatorname{Ind}_{P}^{G}(\sigma), \pi\right) \cong \operatorname{Hom}_{M}\left(\sigma, r_{\bar{P}}^{G}(\pi)\right) .
$$

While the Frobenius reciprocity is an elementary fact, the second form of it is not. If $\pi$ and $\sigma$ are admissible (the case which we shall use), this follows from [C]. For general smooth $\pi$ and $\sigma$, this is proved by J. Bernstein.

For us will be interesting the following case of Frobenius reciprocity. Let $\tau$ and $\omega$ be irreducible representations of $G L(p, F)$ and $S_{q}$ respectively. Let $\pi$ be an admissible representation of $S_{p+q}$. Now Frobenius reciprocity implies

$$
\operatorname{Hom}_{S_{p+q}}(\pi, \tau \rtimes \omega) \cong \operatorname{Hom}_{G L(p, F) \times S_{q}}\left(s_{(p)}(\pi), \tau \otimes \omega\right) .
$$

From the second form of Frobenius reciprocity we get the isomorphism $\operatorname{Hom}_{S_{p+q}}(\tau \rtimes$ $\omega, \pi) \cong \operatorname{Hom}_{G L(p, F) \times S_{q}}\left(\tau \otimes \omega, r_{\bar{P}}^{S_{p+q}}(\pi)\right)$, where $\bar{P}$ denotes the parabolic subgroup opposite to the standard parabolic subgroup which has $G L(p, F) \times S_{q}$ for the Levi subgroup. After conjugation by a suitable element of the Weyl group (which carries $\bar{P}$ to $P$ ), we get

$$
\operatorname{Hom}_{S_{p+q}}(\tau \rtimes \omega, \pi) \cong \operatorname{Hom}_{G L(p, F) \times S_{q}}\left(\tilde{\tau} \otimes \omega, s_{(p)}(\pi)\right) .
$$


Recall that in the above formula $\tau$ must be irreducible. We could write the above formulas in a more general setting, not necessarily for maximal parabolic subgroups.

Let $\rho$ and $\sigma$ be unitarizable irreducible cuspidal representations of a general linear group and of $S_{n}$ respectively. Then if $\nu^{\alpha} \rho \rtimes \sigma$ reduces for some $\alpha \in \mathbb{R}$, then $\rho \cong \tilde{\rho}$. Further, if $\rho \cong \tilde{\rho}$, then we have always reduction for unique $\alpha \geq 0$. This reducibility point will be denoted by

$$
\alpha_{\rho, \sigma}
$$

In all known examples holds

$$
\alpha_{\rho, \sigma} \in(1 / 2) \mathbb{Z}
$$

F. Shahidi has proved this to be the case if $\sigma$ is generic (see [Sh1] and [Sh2]). This is expected to hold in general.

\section{A CRITERION FOR IRREDUCIBILITY}

2.1. Lemma. Let $\pi$ be an irreducible representation of a classical group $S_{q}$ and let $\rho$ be an irreducible cuspidal representation of a general linear group $G L(p, F)$. Suppose

(1) $\rho \neq \tilde{\rho}$.

(2) $\rho \rtimes \pi_{\text {cusp }}$ is irreducible.

(3) $\rho \times \rho^{\prime}$ and $\tilde{\rho} \times \rho^{\prime}$ are irreducible for any factor $\rho^{\prime}$ of $\pi$.

(4) Neither $\rho$ nor $\tilde{\rho}$ is a factor of $\pi$.

Then

$$
\rho \rtimes \pi
$$

is irreducible.

Proof. Observe that if $\rho^{\prime}$ is an irreducible cuspidal representation of a general linear group such that $\rho^{\prime} \otimes \pi^{\prime} \leq \mu^{*}(\pi)$ for some non-zero $\pi$, then $\rho^{\prime}$ is a factor of $\pi$.

Recall that

$$
\mu^{*}(\rho \rtimes \pi)=(1 \otimes \rho+\rho \otimes 1+\tilde{\rho} \otimes 1) \rtimes \mu^{*}(\pi)
$$

Suppose that an irreducible representation $\rho \otimes \tau$ (resp. $\tilde{\rho} \otimes \tau)$ is a subquotient of $\mu^{*}(\rho \rtimes \pi)$. Since $\rho$ (resp. $\tilde{\rho})$ is not a factor of $\pi$, and $\rho \not \tilde{\rho}$, the above formula implies that we can get $\rho \otimes \tau$ (resp. $\tilde{\rho} \otimes \tau)$ only from $(\rho \otimes 1) \rtimes \mu^{*}(\pi)$ (resp. $\left.(\tilde{\rho} \otimes 1) \rtimes \mu^{*}(\pi)\right)$. Considering the grading, we get that $\rho \otimes \tau$ (resp. $\tilde{\rho} \otimes \tau)$ can come only from $(\rho \otimes 1) \rtimes\left(R_{0} \otimes R(S)\right)$ (resp. $\left.(\tilde{\rho} \otimes 1) \rtimes\left(R_{0} \otimes R(S)\right)\right)$. The only term of $\mu^{*}(\rho \rtimes \pi)$ in $R_{0} \otimes R(S)$ is $1 \otimes \pi$. Thus $\rho \otimes \tau \leq(\rho \otimes 1) \rtimes(1 \otimes \pi)=\rho \otimes \pi$ (resp. $\tilde{\rho} \otimes \tau \leq \tilde{\rho} \otimes \pi)$.

The above discussion implies that the multiplicities of $\rho \otimes \pi$ and $\tilde{\rho} \otimes \pi$ in $\mu^{*}(\rho \rtimes \pi)$ are both one. Moreover, we have shown that if $\rho \otimes \tau$ (resp. $\tilde{\rho} \otimes \tau)$ is an irreducible subquotient of $\mu^{*}(\rho \rtimes \pi)$, then it must be $\rho \otimes \pi$ (resp. $\tilde{\rho} \otimes \pi$ ).

By Frobenius reciprocity, any irreducible subrepresentation of $\rho \rtimes \pi$ has $\rho \otimes \pi$ for a quotient of its Jacquet module. Therefore, $\rho \rtimes \pi$ has a unique irreducible subrepresentation. Denote it by $\pi_{s}$. Obviously $\pi_{s}$ can be characterized as an irreducible subquotient of $\rho \rtimes \pi$ which has $\rho \otimes \pi$ for a subquotient of its Jacquet module. 
Take any irreducible quotient of $\rho \rtimes \pi$. Denote it by $\pi_{q}$. Now the second form of Frobenius reciprocity implies that $\tilde{\rho} \otimes \pi$ is a subrepresentation of the Jacquet module of $\pi_{q}$. Therefore, $\rho \rtimes \pi$ has a unique irreducible quotient (and it has $\tilde{\rho} \otimes \pi$ for a subquotient of its Jacquet module). Further, $\pi_{q}$ can be characterized as a subquotient of $\rho \rtimes \pi$ which has $\tilde{\rho} \otimes \pi$ for a subquotient of its Jacquet module).

We know $\pi \hookrightarrow \tau_{1} \times \ldots \times \tau_{\ell} \rtimes \pi_{c u s p}$ for some irreducible cuspidal representations $\tau_{i}$ 's of general linear groups. Then

$$
\pi_{s} \hookrightarrow \rho \rtimes \pi \hookrightarrow \rho \times \tau_{1} \times \ldots \times \tau_{\ell} \rtimes \sigma .
$$

Conditions of the lemma imply, after a series of isomorphisms, $\rho \times \tau_{1} \times \ldots \times \tau_{\ell} \rtimes \sigma \cong$ $\tilde{\rho} \times \tau_{1} \times \ldots \times \tau_{\ell} \rtimes \sigma$. Thus, $\tilde{\rho} \otimes \tau_{1} \otimes \ldots \otimes \tau_{\ell} \otimes \sigma$ is in Jacquet module of $\pi_{s}$. By transitivity of Jacquet modules, it must come from some subquotient in $s_{(p)}\left(\pi_{s}\right)$, which must be of the form $\tilde{\rho} \otimes \alpha$. But a comment at the beginning of proof implies that $\alpha=\pi$, which implies $\pi_{s}=\pi_{q}$. Therefore $\pi_{s}=\pi_{q}=\rho \rtimes \pi$. Thus, we have proved irreducibility in this case.

The above lemma (and the proposition bellow) could be proved by methods of [J] (we expect that more sophisticated methods of $[\mathrm{J}]$ enable the proof of the lemma without assuming (1)).

For a set $X$ of smooth representations, we denote $\tilde{X}=\{\tilde{\pi} ; \pi \in X\}$.

2.2. Proposition. Let $\pi$ be an irreducible representation of a classical group $S_{q}$.

(i) Let $X$ be a set of irreducible cuspidal representations of general linear groups which satisfies

(1) $\nu^{ \pm 1} \rho \notin \tilde{X}$, for any $\rho \in X$.

(2) $X \cap \tilde{X}=\emptyset$.

(3) There is no element in $X \cup \tilde{X}$ which is a factor of $\pi$.

(4) $\rho \rtimes \pi_{\text {cusp }}$ is irreducible for any $\rho \in X$.

(5) $\rho \times \rho^{\prime}$ and $\tilde{\rho} \times \rho^{\prime}$ are irreducible for any $\rho \in X$ and any factor $\rho^{\prime}$ of $\pi$.

Suppose that $\theta$ is an irreducible representation of a general linear group whose cuspidal support is contained in $X$. Then

$$
\theta \rtimes \pi
$$

is irreducible.

(ii) Suppose that we can find sets $X$ and $Y$ of (equivalence classes of) irreducible cuspidal representations of general linear groups such that $X \cup \tilde{X} \cup Y \cup \tilde{Y}$ contains all the factors of $\pi, X \cap(Y \cup \tilde{Y})=\emptyset$, and that hold conditions (1), (2) and (4) from (i). Further suppose that $\rho \times \rho^{\prime}$ and $\tilde{\rho} \times \rho^{\prime}$ are irreducible for all $\rho \in X \cup \tilde{X}$ and $\rho^{\prime} \in Y$ (i.e. that holds condition (5) from (i) for all $\rho \in X \cup \tilde{X}$ and $\rho^{\prime}$ in $Y$ ).

Then there exists an irreducible representation $\theta$ of a general linear whose cuspidal support is contained in $X$ (i.e. each representation of the support), and there exists an irreducible representation $\pi^{\prime}$ of a classical group whose all factors are contained in $Y \cup \tilde{Y}$, such that

$$
\pi \cong \theta \rtimes \pi^{\prime} .
$$

The partial cuspidal support of $\pi^{\prime}$ is $\pi_{\text {cusp. }}$. Further, $\pi$ determines $\theta$ and $\pi^{\prime}$ as above up to equivalence. 
If $X$ is a subset of the set of all the factors of $\pi$, then each representation from $X$ shows up in the cuspidal support of $\theta$.

Proof. (i) Let $\pi, X$ and $\theta$ satisfy conditions of (i). Note that Lemma 2.1 implies that $\varphi \rtimes \pi$ is irreducible for any $\varphi \in X$.

Recall that

$$
\mu^{*}(\theta \rtimes \pi)=M^{*}(\theta) \rtimes \mu^{*}(\pi) .
$$

Suppose that an irreducible representation $\theta \otimes \tau$ (resp. $\tilde{\theta} \otimes \tau)$ is a subquotient of $\mu^{*}(\theta \rtimes \pi)$. Since there does not exist a factor $\rho$ of $\pi$ such that $\rho$ or $\tilde{\rho}$ is in the cuspidal support of $\theta$ (recall that no element of $X \cup \tilde{X}$ is a factor of $\pi$ by (3) and $\theta$ is supported by $X$ ), the above formula implies that we can get $\theta \otimes \tau$ (resp. $\tilde{\theta} \otimes \tau)$ only from $M^{*}(\theta) \rtimes(1 \otimes \pi)$. Considering the grading, we get that $\theta \otimes \tau$ (resp. $\tilde{\theta} \otimes \tau)$ can come only from $M_{G L}^{*}(\theta) \rtimes(1 \otimes \pi)$. Now the formula for $M_{G L}^{*}(\theta)$ from previous section, the condition that $\theta$ is supported by $X$, and $X \cap \tilde{X}=\emptyset$ imply that $\theta \otimes \tau$ (resp. $\tilde{\theta} \otimes \tau)$ can come only from $(\theta \otimes 1) \rtimes(1 \otimes \pi)=\theta \otimes \pi$ (resp. $(\tilde{\theta} \otimes 1) \rtimes(1 \otimes \pi)=\tilde{\theta} \otimes \pi)$.

Thus if $\theta \otimes \tau$ (resp. $\tilde{\theta} \otimes \tau)$ is a subquotient of $\mu^{*}(\theta \rtimes \pi)$, then it must be $\theta \otimes \pi$ (resp. $\tilde{\theta} \otimes \pi$ ). Further, the multiplicity of $\theta \otimes \pi$ (resp. $\tilde{\theta} \otimes \pi$ ) in $\mu^{*}(\pi)$ is 1 . This implies that $\theta \rtimes \pi$ has unique irreducible subrepresentation, which we shall denote by $\pi_{s}$. We can characterize $\pi_{s}$ as unique irreducible subquotient of $\theta \rtimes \pi$ which has $\theta \otimes \pi$ for a subquotient of appropriate Jacquet module. Further, $\theta \rtimes \pi$ has unique irreducible quotient, which we denote by $\pi_{q}$. Similarly as before, $\pi_{q}$ can be characterized as unique irreducible subquotient of $\theta \rtimes \pi$ which has $\tilde{\theta} \otimes \pi$ for a subquotient of a Jacquet module.

Actually, by the argument from the beginning of the proof, we can get more than we stated above. We can conclude the following: suppose that $\theta$ is a representation of $G L(p, F)$ and $\varphi \otimes \tau$ is an irreducible subquotient of $s_{(p)}(\theta \rtimes \pi)$ such that the cuspidal support of $\varphi$ is contained in $X$ (resp. $\tilde{X})$, then $\varphi \otimes \tau=\theta \otimes \pi($ resp. $\varphi \otimes \tau=\tilde{\theta} \otimes \pi$ ).

Let $\varphi \otimes \tau$ be an irreducible subquotient of $s_{(p)}\left(\pi_{s}\right)$, such that $\varphi$ is supported in $X \cup \tilde{X}$, with maximal possible number of representations in $\operatorname{supp}(\varphi)$ (counted with multiplicities), which are in $\tilde{X}$ (note that there is at least one element that satisfies this: $\theta \otimes \pi$ ). If there are no elements of $\operatorname{supp}(\varphi)$ which are in $X$, then they are all in $\tilde{X}$, and thus $\varphi \otimes \tau=\tilde{\theta} \otimes \pi$ by the remark in the above paragraph. Thus $\pi_{s}=\pi_{q}$ by the above characterization of $\pi_{q}$, which implies that $\theta \rtimes \pi$ is irreducible.

Therefore it remains to consider the case where at least one element of $\operatorname{supp}(\varphi)$ is in $X$. Then we can find an irreducible quotient $\varphi_{1} \otimes \tau^{\prime}$ of $s_{(p)}\left(\pi_{s}\right)$, such that the cuspidal support of $\varphi_{1}$ is the same as of $\varphi$ (this follows easily using decomposition determined by infinitesimal characters). By Frobenius reciprocity, $\pi_{s} \hookrightarrow \varphi_{1} \rtimes \tau^{\prime}$. Write $\varphi_{1}=\varphi_{-} \times \varphi_{+}$, where the cuspidal support of $\varphi_{+}\left(\right.$resp. $\left.\varphi_{-}\right)$is contained in $X($ resp. $\tilde{X})$. We can do this, according to assumptions of the lemma (use assumption (1)).

We know $\varphi_{+} \hookrightarrow \tau_{1} \times \ldots \times \tau_{l-1} \times \tau_{\ell}$, for some $\tau_{i} \in X$ and $\ell \geq 1$. This implies that we have an embedding $\pi_{s} \hookrightarrow \varphi_{-} \times \tau_{1} \times \ldots \times \tau_{\ell} \rtimes \tau^{\prime}$. Note that $\tau_{\ell} \rtimes \tau^{\prime}$ is irreducible by Lemma 2.1 (observe that $\tau_{\ell}$ and $\tau^{\prime}$ satisfy conditions of Lemma 2.1). Therefore $\tau_{1} \times \ldots \times \tau_{\ell-1} \times \tau_{\ell} \rtimes \tau^{\prime} \cong$ $\tau_{1} \times \ldots \times \tau_{\ell-1} \times \tilde{\tau}_{\ell} \rtimes \tau^{\prime}$.

Thus $\pi_{s} \hookrightarrow \varphi_{-} \times \tau_{1} \times \ldots \times \tau_{\ell-1} \times \tilde{\tau}_{\ell} \rtimes \tau^{\prime}$, which implies (using Frobenius reciprocity) that $\varphi_{-} \otimes \tau_{1} \otimes \ldots \otimes \tau_{\ell-1} \otimes \tilde{\tau}_{\ell} \otimes \tau^{\prime}$ is a quotient of appropriate Jacquet module of $\pi_{s}$. 
By the transitivity of Jacquet modules, we must be able to get it from an irreducible subquotient of $s_{(p)}\left(\pi_{s}\right)$, say from $\varphi_{2} \otimes \tau^{\prime \prime}$. Note that the support of $\varphi_{2}$ is again contained in $X \cup \tilde{X}$, and further, that the cuspidal support of $\varphi_{2}$ has one representation more in $\tilde{X}$ then the cuspidal support of $\varphi_{1}$, and therefore then the cuspidal support of $\varphi$. This is a contradiction, which completes the proof of (i).

(ii) Suppose that $\pi, X$ and $Y$ satisfy the conditions of (ii). The representation $s_{G L}(\pi)$ has some irreducible quotient. Denote such a quotient by

$$
\pi_{1} \otimes \pi_{\text {cusp }}
$$

Now the cuspidal support of $\pi_{1}$ is contained in $X \cup \tilde{X} \cup Y \cup \tilde{Y}$. Conditions on $X$ and $Y$ imply that there exist irreducible representations $\pi_{X}$ and $\pi_{Y}$ such that $\pi=\pi_{X} \times \pi_{Y}$ and that the cuspidal support of $\pi_{X}$ is contained in $X \cup \tilde{X}$ and of $\pi_{Y}$ in $Y \cup \tilde{Y}$. Now Frobenius reciprocity implies that $\pi$ is a subrepresentation of

$$
\pi_{X} \times \pi_{Y} \rtimes \pi_{c u s p} .
$$

Therefore, there exists an irreducible subquotient $\sigma_{Y}$ of $\pi_{Y} \times \pi_{\text {cusp }}$ such that $\pi$ is subquotient of

$$
\pi_{X} \rtimes \sigma_{Y} .
$$

Clearly, all the factors of $\sigma_{Y}$ are contained in $Y \cup \tilde{Y}$.

We can write $\pi_{X}=\pi_{X}^{+} \times \pi_{X}^{-}$in a way that cuspidal supports of $\pi_{X}^{+}$and $\pi_{X}^{-}$are contained in $X$ and $\tilde{X}$ respectively (use conditions (1) and (2)). Since $\pi_{X}^{-} \rtimes \sigma_{Y}$ and $\left(\pi_{X}^{-}\right)^{\sim} \rtimes \sigma_{Y}$ have the same composition series, $\pi$ is a subquotient of

$$
\pi_{X}^{+} \times\left(\pi_{X}^{-}\right)^{\sim} \rtimes \sigma_{Y}
$$

Now there exists an irreducible subquotient $\pi_{X}^{\prime}$ of $\pi_{X}^{+} \times\left(\pi_{X}^{-}\right)^{\sim}$ such that $\pi$ is a subquotient of

$$
\pi_{X}^{\prime} \rtimes \sigma_{Y} .
$$

Clearly, the support of $\pi_{X}^{\prime}$ is contained in $X$. Now from (i) we get that $\pi_{X}^{\prime} \rtimes \sigma_{Y}$ is irreducible. Thus $\pi \cong \pi_{X}^{\prime} \rtimes \sigma_{Y}$. This proves the existence of decomposition in (ii).

Suppose that $\theta^{\prime} \rtimes \pi^{\prime}$ and $\theta^{\prime \prime} \rtimes \pi^{\prime \prime}$ are two decompositions of $\pi$ considered in (ii). Recall that if $\pi$ is parabolically induced by two irreducible cuspidal representations, then they must be conjugated by the Weyl group. The action of the Weyl group and conditions on $X$ and $Y$ imply that $\theta^{\prime}$ and $\theta^{\prime \prime}$ are representations of the same general linear group. Denote it by $G L(l, F)$. Moreover, the representations $\theta$ and $\theta^{\prime}$ must have the same cuspidal support. Frobenius reciprocity implies that $\theta^{\prime \prime} \otimes \pi^{\prime \prime}$ is a subquotient $s_{(l)}(\pi)=s_{(l)}\left(\theta^{\prime} \rtimes \pi^{\prime}\right)$. Now the first part of the proof of $(i)$ implies that $\theta^{\prime} \otimes \pi^{\prime} \cong \theta^{\prime \prime} \otimes \pi^{\prime \prime}$, which implies $\theta^{\prime} \cong \theta^{\prime \prime}$ and $\pi^{\prime} \cong \pi^{\prime \prime}$.

\section{Some CONSEquences}

J. Bernstein defined rigid representations of general linear groups in [Bn]. We shall recall his definition, and extend it to the classical groups. 


\subsection{Definitions.}

(i) Let $\tau$ be an irreducible representation of a general linear group. Then $\tau$ called rigid if each $\rho$ in the cuspidal support of $\tau$ satisfies

$$
e(\rho) \in(1 / 2) \mathbb{Z}
$$

Further, $\tau$ is called strongly rigid, if it is rigid, and if it satisfy the following condition: if $\rho, \rho^{\prime}$ are in the cuspidal support of $\tau$ and $\rho^{u} \cong\left(\rho^{\prime}\right)^{u}$, then

$$
e(\rho)-e\left(\rho^{\prime}\right) \in \mathbb{Z}
$$

(ii) An irreducible representation $\pi$ of $S_{n}$ will be called weakly real if

$$
\tilde{\rho^{u}} \cong \rho^{u}
$$

for any factor $\rho$ of $\pi$. It will be called rigid if it is weakly real and if

$$
e(\rho) \in(1 / 2) \mathbb{Z}
$$

for any factor $\rho$ of $\pi$. Further, it will be called strongly rigid if it is weakly real and

$$
e(\rho)+\alpha_{\rho^{u}, \pi_{\text {cusp }}} \text { and } e(\rho)-\alpha_{\rho^{u}, \pi_{\text {cusp }}} \in \mathbb{Z}
$$

for any factor $\rho$ of $\pi$.

Note that if $\pi$ is strongly rigid, it is rigid. Further, $\rho^{u}$ and $\pi_{c u s p}$ satisfy (HI) for any factor $\rho$ of a strongly rigid representation $\pi$.

The Hermitian contragredient of a representation $\pi$ will be denoted by $\pi^{+}$(i.e. $\pi^{+}$ is the complex conjugate of $\tilde{\pi})$. Representation $\pi$ is called Hermitian if $\pi \cong \pi^{+}$. Each admissible unitarizable representation is Hermitian.

From the Langlands classification (see the following section), and the formula for the contragredient in it (see [T5]), follow that each weakly real representation is Hermitian.

Denote by $\mathcal{C}$ the set of all the equivalence classes of irreducible cuspidal representations of general linear groups $G L(n, F), n \geq 1$. The subset of the unitarizable classes is denoted by $\mathcal{C}_{u}$.

3.2. Theorem. Let $\pi$ be an irreducible representation of $S_{n}$.

(i) Suppose that $\pi$ is unitarizable. Then there exist an irreducible unitarizable representation $\theta$ of a general linear group and a weakly real irreducible unitarizable representation $\pi^{\prime}$ of some $S_{n^{\prime}}$, such that

$$
\pi \cong \theta \rtimes \pi^{\prime}
$$

(ii) Let $\mathcal{C}_{u}^{\prime}$ be a subset of $\mathcal{C}_{u}$ satisfying $\mathcal{C}_{u}^{\prime} \cap \tilde{\mathcal{C}}_{u}^{\prime}=\emptyset$, such that $\mathcal{C}_{u}^{\prime} \cup \tilde{\mathcal{C}}_{u}^{\prime}$ contains all $\rho \in \mathcal{C}_{u}$ which are not self dual. Denote

$$
\mathcal{C}^{\prime}=\left\{\nu^{\alpha} \rho ; \alpha \in \mathbb{R}, \rho \in \mathcal{C}_{u}^{\prime}\right\}
$$


Then there exists an irreducible representation $\theta$ of a general linear group with support contained in $\mathcal{C}^{\prime}$, and a weakly real irreducible representation $\pi^{\prime}$ of some $S_{n^{\prime}}$ such that

$$
\pi \cong \theta \rtimes \pi^{\prime} .
$$

Moreover, $\pi$ determines such $\theta$ and $\pi^{\prime}$ up to an equivalence. Further, $\pi$ is unitarizable (resp. Hermitian) if and only both $\theta$ and $\pi^{\prime}$ are unitarizable (resp. Hermitian).

Proof. Clearly, one can find a set $\mathcal{C}_{u}^{\prime}$ which satisfies the conditions in (ii). Obviously, (ii) implies (i). Therefore, it enough to prove (ii).

Denote by $X$ the set $\mathcal{C}$ from (ii) and let $Y_{u}$ be the set of all self dual representations in $\mathcal{C}$. Let $Y=\left\{\nu^{\alpha} \rho ; \alpha \in \mathbb{R}, \rho \in Y_{u}\right\}$. Obviously, $X$ and $Y$ satisfy (ii) of Proposition 2.2. Applying this proposition, we get the decomposition of $\pi$, and the unicity of the decomposition.

Obviously, if $\theta$ and $\pi^{\prime}$ are unitarizable (resp. Hermitian), then $\pi$ is unitarizable (resp. Hermitian).

Suppose that $\pi=\theta \rtimes \pi^{\prime}$ is Hermitian. Then $\theta \rtimes \pi^{\prime} \cong \theta^{+} \rtimes\left(\pi^{\prime}\right)^{+}$. Now Frobenius reciprocity implies that $\theta^{+} \otimes\left(\pi^{\prime}\right)^{+}$is a subquotient of a Jacquet module of $\pi=\theta \rtimes \pi^{\prime}$. Note that $\theta^{+}$is supported again in $X$. Similarly as in the proof of unicity in (ii) follows that cuspidal supports of $\theta$ and $\theta^{+}$must be the same. Now the first part of the proof of (i) of Proposition 2.1 implies $\theta^{+} \otimes\left(\pi^{\prime}\right)^{+} \cong \theta \otimes \pi^{\prime}$, which implies $\theta^{+} \cong \theta$ and $\left(\pi^{\prime}\right)^{+} \cong \pi^{\prime}$, i.e. $\theta$ and $\pi^{\prime}$ are Hermitian.

Suppose now that $\pi$ is unitarizable. Then it is Hermitian. The above part of the proof implies that $\theta \otimes \pi^{\prime}$ is Hermitian. Now we know that $\theta \otimes \pi^{\prime}$ is unitarizable (see for example construction (d) in the third section of [T6]). This implies that $\theta$ and $\pi^{\prime}$ are unitarizable. The proof of (ii) is now complete.

Since we have a classification of irreducible unitarizable representations of general linear groups, the above theorem reduces the classification of irreducible unitarizable representations of groups $S_{n}$ to the classification of weakly real irreducible unitarizable representations of groups $S_{n}$.

Let $Z \subseteq \mathcal{C}_{u}$. Denote by

$$
\operatorname{Irr}_{Z}^{\mathbb{R}}
$$

the set of all equivalence classes of irreducible representations of general linear groups supported in $\left\{\nu^{\alpha} \rho ; \alpha \in \mathbb{R}\right\}$. For $Z \subseteq \mathcal{C}_{u}$ and an irreducible cuspidal representation $\sigma$ of $S_{m}$, let

$$
\mathcal{I}(Z ; \sigma)
$$

denote the set of all equivalence classes of irreducible representations $\pi$ of groups $S_{n}$ 's for which we can find $\alpha_{1}, \ldots, \alpha_{k} \in \mathbb{R}$ and $\rho_{1}, \ldots, \rho_{k} \in Z$ such that $\pi$ is isomorphic to a subquotient of $\nu^{\alpha_{1}} \rho_{1} \times \ldots \times \nu^{\alpha_{k}} \rho_{k} \rtimes \sigma$. We shall point out two interesting consequences of the above theorem.

3.2. Corollary. Let $\sigma$ be an irreducible cuspidal representation of $S_{m}$.

(i) Let $\mathcal{C}_{u}^{\prime}$ be as in (ii) of the above theorem. Then

$$
\theta \mapsto \theta \rtimes \sigma
$$


defines a bijection of $\operatorname{Irr}_{\mathcal{C}_{u}^{\prime}}^{\mathbb{R}}$ onto $\mathcal{I}\left(\mathcal{C}_{u}^{\prime} ; \sigma\right)$, and

$$
\theta \text { is unitarizable } \Longleftrightarrow \theta \rtimes \sigma \text { is unitarizable. }
$$

(ii) Let $\rho \in \mathcal{C}_{u}, \rho \neq \tilde{\rho}$. Then $\theta \mapsto \theta \rtimes \sigma$ defines a bijection of $\operatorname{Irr}_{\rho}^{\mathbb{R}}$ onto $\mathcal{I}(\rho ; \sigma)$. Moreover, $\theta$ is unitarizable $\Longleftrightarrow \theta \rtimes \sigma$ is unitarizable.

In particular, in this way we get reduction of unitarizability in $\mathcal{I}(\rho ; \sigma)$ to the well known case of general linear groups.

Proof. (i) Mapping $\theta \mapsto \theta \rtimes \sigma$ carries $\operatorname{Irr}_{\mathcal{C}_{u}^{\prime}}^{\mathbb{R}}$ to $\mathcal{I}\left(\mathcal{C}_{u}^{\prime} ; \sigma\right)$ since $\theta \rtimes \sigma$ is irreducible by (i) of Proposition 2.2. From (ii) of the same proposition follows that it is surjective. To see injectivety, suppose $\theta \rtimes \sigma \cong \theta^{\prime} \rtimes \sigma$. Then $\theta \otimes \sigma$ is a subquotient of the Jacquet module of $\theta^{\prime} \rtimes \sigma$. Now the first part of the proof of (i) of Proposition 2.2 implies $\theta \cong \theta^{\prime}$. Unitarizability claim follows in the same way as in the proof of the above theorem (first one proves that $\theta$ is Hermitian if and only if $\theta \rtimes \sigma$ is Hermitian; note that cuspidal $\sigma$ is always unitarizable and Hermitian).

One proves (ii) in the same way.

3.3. Remarks. Take $\rho_{1}, \ldots, \rho_{k} \in \mathcal{C}_{u}$ such that sets $\left\{\rho_{i}, \tilde{\rho}_{i}\right\}, 1 \leq i \leq k$, have two elements each, and that these sets are different.

(i) One gets easily, using arguments from the proof of above theorem (and basic properties of the representation theory of general linear groups), that $\left(\theta_{1}, \ldots, \theta_{k}\right) \mapsto \theta_{1} \times \ldots \times \theta_{k} \rtimes \sigma$ defines a bijection of $\operatorname{Irr}_{\rho_{1}}^{\mathbb{R}} \times \ldots \times \operatorname{Irr} r_{\rho_{1}}^{\mathbb{R}}$ onto $\mathcal{I}\left(\rho_{1}, \ldots, \rho_{k} ; \sigma\right)$, and that this bijection preserves the unitarizability in both directions.

(ii) By (ii) of the above corollary, $\left(\theta_{1}, \ldots, \theta_{k}\right) \mapsto\left(\theta_{1} \rtimes \sigma, \ldots, \theta_{k} \rtimes \sigma\right)$ defines a bijection of $\operatorname{Irr}_{\rho_{1}}^{\mathbb{R}} \times \ldots \times \operatorname{Irr} r_{\rho_{1}}^{\mathbb{R}}$ onto $\mathcal{I}\left(\rho_{1} ; \sigma\right) \times \ldots \times \mathcal{I}\left(\rho_{k} ; \sigma\right)$, which preserves the unitarizability (in both directions). Combining this bijection with the bijection from (i), we get a bijection

$$
\begin{aligned}
\left(\pi_{1}, \ldots, \pi_{k}\right) & \mapsto \pi, \\
I\left(\rho_{1} ; \sigma\right) \times \ldots \times \mathcal{I}\left(\rho_{k} ; \sigma\right) & \rightarrow I\left(\rho_{1}, \ldots, \rho_{k} ; \sigma\right) .
\end{aligned}
$$

This bijection satisfies

$\pi$ is unitarizable if and only if $\pi_{i}$ are unitarizable for all $1 \leq i \leq k$.

(iii) One sees easily that the bijection in (i) depends on the fact that $\rho_{i}$ 's are not self dual. It is hard to expect that such bijection can be established in the self dual case, in particular, in a way that the unitarizability is preserved in both directions (because if $\rho$ is self dual, we have much more unitarizable representations in $\mathcal{I}(\rho ; \sigma)$ than in $\left.\operatorname{Irr} r_{\rho}^{\mathbb{R}}\right)$. From the other side, the bijection in (ii) can be extended to the general case. C. Jantzen has defined such a bijection in general case in $[\mathrm{J}]$ (without assuming that $\rho_{i}$ are not self dual). We expect that this bijection in general preserves the unitarizability. This would reduce the problem of unitarizability to the same problem in "lines" $I(\rho ; \sigma)$. Recall that (ii) of the above corollary solves the problem of unitarizability in the (easy) case of a line $I(\rho ; \sigma)$, when $\rho \neq \tilde{\rho}$.

The following proposition is useful for constructing complementary series. 
3.4. Lemma. Let $\tau$ and $\pi$ be irreducible representations of a general linear group and $S_{m}$ respectively. Suppose that $\tau$ is strongly rigid and that (HI) holds. Let $\rho^{u} \cong \tilde{\rho}^{u}$ for each $\rho$ in the cuspidal support of $\tau$. If $\pi$ is rigid, then representations $\nu^{\alpha} \tau \rtimes \pi, \alpha \in \mathbb{R} \backslash(1 / 2) \mathbb{Z}$, are irreducible.

Under the assumptions of the above lemma, using $[\mathrm{M} \mathrm{T}]$ we can improve the above result if $\tau \rtimes \pi$ contains a strongly rigid subquotient (but we need to assume that (BA) from $[\mathrm{M} T$ T holds).

Proof. Denote by $X_{0}$ the set of all representations which are in the support of $\tau$. Fix $\alpha$ in $\mathbb{R} \backslash(1 / 2) \mathbb{Z}$. Let $X=\left\{\nu^{\alpha} \rho ; \rho \in X_{0}\right\}$. Now one directly checks that the conditions (1)(5) in (i) of Proposition 2.2 are satisfied (use that $\pi$ is rigid, $\tau$ strongly rigid and that we assume (HI)). Proposition 2.2 implies the irreducibility.

Theorem 3.1 reduces the unitarizability problem to the weakly real case. The following proposition provides an upper bound for unitarizability in the weakly real case.

3.5. Proposition. Let $\pi$ be an irreducible unitarizable representation of a classical group $S_{q}$. Let $\rho$ be a factor of $\pi$ such that

$$
\rho^{u} \cong \widetilde{\rho^{u}}
$$

Suppose that $\rho_{1}, \ldots, \rho_{n}$ are all the factors $\tau$ of $\pi$ such that $\tau^{u} \cong \rho^{u}$. Write

$$
\left\{\left|e\left(\rho_{i}\right)\right| ;\left|e\left(\rho_{i}\right)\right|>\alpha_{\rho^{u}, \pi_{c u s p}}\right\}=\left\{\alpha_{1}, \ldots, \alpha_{\ell}\right\},
$$

where $\ell \geq 0$ and $\alpha_{1}<a_{2}<\cdots<\alpha_{\ell}$. Then

(i) $\alpha_{1}-\alpha_{\rho^{u}, \pi_{c u s p}} \leq 1$ if $\ell \geq 1$, and

$$
\alpha_{i}-\alpha_{i-1} \leq 1 \quad \text { for each } i=2,3, \ldots, \ell .
$$

$$
\alpha_{i} \leq \alpha_{\rho^{u}, \pi_{\text {cusp }}}+i ; \quad i=1, \ldots, \ell .
$$

Proof. Obviously, it is enough to prove (i). After modifying enumeration, we can assume $\left|e\left(\rho_{1}\right)\right| \leq \cdots \leq\left|e\left(\rho_{n}\right)\right|$. Suppose that we have a gap greater than 1 . It must be before some $\left|e\left(\rho_{i}\right)\right|$. Fix one such $i$ and take minimal $i_{0}$ such that $\left|e\left(\rho_{i_{0}}\right)\right|=\left|e\left(\rho_{i}\right)\right|$. By Proposition 2.2, we can write $\pi=\tau \rtimes \pi^{\prime}$, where the cuspidal support of $\tau$ is contained in $\left\{\rho_{i_{0}}^{\uparrow}, \ldots, \rho_{n}^{\uparrow}\right\} \cup$ $\left\{\left(\rho_{i_{0}}^{\uparrow}\right)^{\sim}, \ldots,\left(\rho_{n}^{\uparrow}\right)^{\sim}\right\}=\left\{\rho_{i_{0}}, \ldots, \rho_{n}\right\} \cup\left\{\tilde{\rho}_{i_{0}}, \ldots, \tilde{\rho}_{n}\right\}$ and no one of these representations or their contragredients is a factor of $\pi^{\prime}$. Moreover, if $\nu^{\alpha} \rho_{u}$ is a factor of $\pi^{\prime}$, then $|\alpha|<$ $\left|e\left(\rho_{i}\right)\right|-1$ (this follows from the existence of the gap).

Note that by the same proposition, $\nu^{\alpha} \tau \rtimes \pi^{\prime}$ is irreducible for $\alpha \geq 0$.

From unitarizability of $\pi$ follows that $\pi^{\prime}$ is Hermitian (use the formula for the contragredient in the Langlands classification from [T5]). Now representations $\nu^{\alpha} \tau \rtimes \pi^{\prime}$ form a continuous family of Hermitian representations, with one unitarizable representation in it (see [T6] for the definition of such a family). Then all the representations in the family are unitarizable (see the construction (b) from the third section of [T6]). This is impossible since this family is not bounded (see [T3]). 


\section{LANGLANDS PARAMETER OF A SUBQUOTIENT}

Denote by $D$ the set of all classes of essentially square integrable representations of all $G L(n, F)$ 's, $n \geq 1$. The set of all finite multisets in $D$ is denoted by $M(D)$. Let $d=\left(\delta_{1}, \ldots, \delta_{l}\right) \in M(D)$. Take a permutation $p$ of $\{1, \ldots, l\}$ such that

$$
e\left(\delta_{p(1)}\right) \geq \cdots \geq e\left(\delta_{p(l)}\right)
$$

If $p=i d$ satisfies this condition, then we say that $d=\left(\delta_{1}, \ldots, \delta_{l}\right)$ is written in a standard order. The representation

$$
\lambda(d)=\delta_{p(1)} \times \ldots \times \delta_{p(l)}
$$

has a unique irreducible quotient, which will be denoted by $L(d)$. This is Langlands classification of irreducible representations of general linear groups. The multiplicity of $L(d)$ in $\delta_{p(1)} \times \ldots \times \delta_{p(l)}\left(\right.$ and in $\left.\delta_{1} \times \ldots \times \delta_{l}\right)$ is 1 .

Let $\delta_{1}, \delta_{2} \in D$. Suppose that $\delta_{1} \times \delta_{2}$ reduces. Then it is a multiplicity one representation of length 2 . One subquotient is $L\left(\delta_{1}, \delta_{2}\right)$ while the other is of the form

$$
\delta_{1,2}^{\cup} \times \delta_{1,2}^{\bigcap}
$$

for unique representations $\delta_{1,2}^{\cup}, \delta_{1,2}^{\cap} \in D$ of $G L\left(n_{1}\right)$ and $G L\left(n_{2}, F\right)$ respectively, such that $n_{1}>n_{2}$ (see $[\mathrm{Z}]$ for details). In the case of reducibility we have

$$
\min \left\{e\left(\delta_{1}\right), e\left(\delta_{2}\right)\right\}<e\left(\delta_{1,2}^{\cup}\right), e\left(\delta_{1,2}^{\cap}\right)<\max \left\{e\left(\delta_{1}\right), e\left(\delta_{2}\right)\right\} .
$$

We shall recall of a well known property of the Langlands classification (which follows from the factorization of so called long intertwining operator in the Langlands classification). If $\pi$ is an irreducible subquotient of $\delta_{p(1)} \times \ldots \times \delta_{p(l)}\left(\right.$ or $\left.\delta_{1} \times \ldots \times \delta_{l}\right)$ different from $L(d)$, then there exists $1 \leq i<j \leq l$ such that $\delta_{i} \times \delta_{j}$ reduces and $\pi$ is a subquotient of

$$
\delta_{1} \times \delta_{2} \times \ldots \times \delta_{i-1} \times \delta_{i, j}^{\cup} \times \delta_{i+1} \times \ldots \times \delta_{j-1} \times \delta_{i, j}^{\cap} \times \delta_{j+1} \times \ldots \times \delta_{l-1} \times \delta_{l} .
$$

We can write

$$
L\left(\delta_{1}, \ldots, \delta_{l}\right)=\delta_{1} \times \ldots \times \delta_{l}+\sum_{c \in X_{d}} m_{d, c} L(c)
$$

in the Grothendieck group, where $X_{d}$ is a finite subset of $M(D)$ such that $m_{d, c} \neq 0$ for all $c \in X_{d}$ (these conditions uniquely determine $X_{d}$; note that $m_{d, c}$ are negative). Clearly $d \notin X_{d}$. From the above property follows that if $c \in X_{d}$, then $L(c)$ is a subquotient of (4-2) for some $i$ and $j$ as above.

Now we shall recall the Langlands classification for groups $S_{k}$. Let

$$
D_{+}=\{\delta \in D ; e(\delta)>0\} .
$$

Denote by $T(S)$ the set of all classes of irreducible tempered representations of groups $S_{k}, k \geq 0$. We shall write an element $t=\left(\left(\gamma_{1}, \ldots, \gamma_{m}\right), \tau\right) \in M\left(D_{+}\right) \times T(S)$ simply as $\left(\gamma_{1}, \ldots, \gamma_{m} ; \tau\right)$. Take a permutation $p$ of $\{1, \ldots, m\}$ such that $e\left(\gamma_{p(1)}\right) \geq \cdots \geq e\left(\gamma_{p(m)}\right)$ (if 
$p=i d$ satisfies this condition, then we say that $t=\left(\gamma_{1}, \ldots, \gamma_{m} ; \tau\right)$ is written in a standard order). Then the representation

$$
\gamma_{p(1)} \times \gamma_{p(2)} \times \ldots \times \gamma_{p(m)} \rtimes \tau
$$

has a unique irreducible quotient, which is denoted by $L(t)$. This is the Langlands classification for groups $S_{k}$ 's. The multiplicity of $L(t)$ in (4-3) is one.

Now we shall recall a property of irreducible subquotients of (4-3), similar to the property that we have described in the case of general linear groups. We can write

$$
L\left(\gamma_{1}, \ldots, \gamma_{m} ; \tau\right)=\gamma_{1} \times \ldots \times \gamma_{m} \rtimes \tau+\sum_{s \in Y_{t}} n_{t, s} L(s)
$$

in the Grothendieck group, where $n_{t, s} \neq 0$ for all $s$ from a finite subset $Y_{t} \subseteq M\left(D_{+}\right) \times T(S)$ (actually, $n_{t, s}$ are negative). Again $t \notin Y_{t}$. Further, for each $L(s), s \in Y_{t}$ at least one of the following two claims hold:

(4-4) there exist $1 \leq i<j \leq m$ and $\varepsilon_{i} \in\{ \pm 1\}$ such that $\left(\gamma_{i}\right)^{\varepsilon_{i}} \times \gamma_{j}$ reduces and that $L(s)$ is subquotient of

$\gamma_{1} \times \ldots \times \gamma_{i-1} \times\left(\gamma_{i, j, \varepsilon_{i}}\right)^{\cap} \times \gamma_{i+1} \times \ldots \times \gamma_{j-1} \times\left(\gamma_{i, j, \varepsilon_{i}}\right)^{\cup} \times \gamma_{j+1} \times \ldots \times \gamma_{m} \rtimes \tau$,

where $\left(\gamma_{i}\right)^{\varepsilon_{i}}$ denotes $\gamma_{i}$ if $\varepsilon_{1}=1$ and $\left(\gamma_{i}\right)^{\varepsilon_{i}}$ denotes $\tilde{\gamma}_{i}$ if $\varepsilon_{i}=-1$, and $\left(\gamma_{i, j, \varepsilon_{i}}\right)^{\cap} \times$ $\left(\gamma_{i, j, \varepsilon_{i}}\right)^{\cup}$ is subquotient of $\left(\gamma_{i}\right)^{\varepsilon_{i}} \times \gamma_{j}$ different from $L\left(\left(\gamma_{i}\right)^{\varepsilon_{i}}, \gamma_{j}\right)$;

or

(4-5) there exists $1 \leq i \leq m$ such that $\gamma_{i} \rtimes \tau$ reduces and if we write $\gamma_{i} \rtimes \tau=L\left(\gamma_{i} ; \tau\right)+$ $K\left(\gamma_{i} ; \tau\right)$ in the Grothendieck group, then $L(s)$ is subquotient of

$$
\gamma_{1} \times \ldots \times \gamma_{i-1} \times \gamma_{i+1} \times \ldots \times \gamma_{m} \rtimes K\left(\gamma_{i} ; \tau\right) .
$$

We could describe subquotients in (4-4) by two $\varepsilon_{i}$ and $\varepsilon_{j}$ from $\{ \pm 1\}$, but the subquotients that we would get in the remaining cases are already covered by (4-4).

We shall now recall of an inequality which must satisfy $\pi=L(s)$ if it is an irreducible subquotient of (4-3) $\left(s \in M\left(D_{+}\right) \times T(S)\right)$. Suppose that (4-3) is a representation of $S_{k}$. First we introduce a partial order on $\mathbb{R}^{k}$ defined by

$$
\begin{aligned}
\left(x_{1}, \ldots, x_{k}\right) & \leq\left(y_{1}, \ldots, y_{k}\right) \Leftrightarrow \\
x_{1} & \leq y_{1}, \\
x_{1}+x_{2} & \leq y_{1}+y_{2}, \\
& \vdots \\
x_{1}+\ldots+x_{k} & \leq y_{1}+\ldots+y_{k} .
\end{aligned}
$$


Now we shall define $\mathbf{e}(t)$ (analogously one defines $\mathbf{e}(s)$ ). Suppose that $\gamma_{p(i)}$ are representations of $G L\left(k_{i}, F\right)$, and $\tau$ is a representation of $S_{l}$. For $p$ is as in (4-3), set

$$
\mathbf{e}(t)=(\underbrace{e\left(\gamma_{p(1)}\right), \ldots, e\left(\gamma_{p(1)}\right)}_{k_{1} \text { times }}, \ldots, \underbrace{e\left(\gamma_{p(n)}\right), \ldots, e\left(\gamma_{p(n)}\right)}_{k_{m} \text { times }}, \underbrace{0,0, \ldots, 0}_{l \text { times }}) .
$$

If $L(s)$ is a subquotient of $\gamma_{1} \times \ldots \times \gamma_{m} \rtimes \tau\left(t\right.$ is $\left.\left(\gamma_{1}, \ldots, \gamma_{m} ; \tau\right)\right)$ such that $s \neq t$, then

$$
\mathbf{e}(s)<\mathbf{e}(t) .
$$

An irreducible representation $\pi$ of $S_{k}$ is equivalent to $L(s)$, for some $s \in M\left(D_{+}\right) \times T(S)$. We define $\mathbf{e}(\pi)$ to be $\mathbf{e}(s)$.

4.1. Remark. If $\sigma$ is a subquotient of some of the representations in (4-4) or (4-5), then

$$
\mathbf{e}(\pi)<\mathbf{e}(t) .
$$

More details and explanation regarding the above properties can be found in [T5].

For $\delta \in D$ which is not unitarizable, denote

$$
\delta^{\uparrow}=\left\{\begin{array}{lll}
\delta & \text { if } & e(\delta)>0 \\
\tilde{\delta} & \text { if } & e(\delta)<0 .
\end{array}\right.
$$

Let $d \in M(D)$. By

$$
d^{\uparrow}
$$

we shall denote the element of $M\left(D_{+}\right)$which we get from $d$ by removing all the unitarizable $\delta_{i}$ 's, and changing all $\delta_{i}$ 's for which $e\left(\delta_{i}\right)<0$, by $\tilde{\delta}_{i}=\delta_{i}^{\uparrow}$. Denote by

$$
d_{u}
$$

the multiset in $M(D)$ which we get from $d$ removing all $\delta_{i}$ 's which are not unitarizable.

We add multisets in obvious way: $\left(x_{1}, \ldots, x_{n}\right)+\left(y_{1}, \ldots, y_{m}\right)=\left(x_{1}, \ldots, x_{n}, y_{1}, \ldots, y_{m}\right)$.

4.2. Proposition. Let $d \in M(D)$ and $t=\left(d^{\prime}, \tau\right) \in M\left(D_{+}\right) \times T(S)$. Denote by

$$
T_{d, \tau}
$$

the set of all (equivalence classes of) irreducible subrepresentations of

$$
\lambda\left(d_{u}\right) \rtimes \tau .
$$

Then:

(i) Each of the representations

$$
L\left(d^{\uparrow}+d^{\prime} ; \tau^{\prime}\right), \quad \tau^{\prime} \in T_{d, \tau}
$$


is a subquotient of

$$
L(d) \rtimes L\left(d^{\prime} ; \tau\right)
$$

(ii) Suppose that $F$ is a field of characteristic 0. Then the multiplicity of each of $L\left(d^{\uparrow}+\right.$ $\left.d^{\prime} ; \tau^{\prime}\right), \tau^{\prime} \in T_{d, \tau}$, in $L(d) \rtimes L\left(d^{\prime} ; \tau\right)$ is one.

Proof. Take $\delta_{1}, \ldots, \delta_{l} \in D, \gamma_{1}, \ldots, \gamma_{m} \in D_{+}$and $\tau \in T(S)$ such that $d=\left(\delta_{1}, \ldots, \delta_{l}\right)$ and $\delta^{\prime}=\left(\gamma_{1}, \ldots, \gamma_{m}\right)$. After modifying enumeration we can assume that $e\left(\delta_{i}\right) \neq 0$ for $1 \leq i \leq n$ and $e\left(\delta_{i}\right)=0$ for $n+1 \leq i \leq l$, for some $0 \leq n \leq l$. Write

$$
\delta_{n+1} \times \ldots \times \delta_{l} \rtimes \tau=\oplus_{\tau^{\prime} \in T_{d, \tau}} k_{\tau^{\prime}} \tau^{\prime}
$$

where $k_{\tau^{\prime}}$ are positive integers.

For the proof of the first claim of the proposition, we need to prove that each representation from the set

$$
\left\{L\left(\delta_{1}^{\uparrow}, \ldots, \delta_{n}^{\uparrow}, \gamma_{1}, \ldots, \gamma_{m} ; \tau^{\prime}\right) ; \tau^{\prime} \in T_{d, \tau}\right\}
$$

is a subquotient of

$$
L\left(\delta_{1}, \ldots, \delta_{l}\right) \rtimes L\left(\gamma_{1}^{\prime}, \ldots, \gamma_{m}^{\prime} ; \tau\right),
$$

and for the second claim of the proposition that the multiplicity of each of these subquotients in (4-8) is one.

First observe that we have in the Grothendieck group

$$
\begin{gathered}
L(d) \rtimes L(t)=L(d) \rtimes L\left(d^{\prime} ; \tau\right)=L\left(\delta_{1}, \ldots, \delta_{l}\right) \rtimes L\left(\gamma_{1}, \ldots, \gamma_{m} ; \tau\right) \\
=\left(\delta_{1} \times \ldots \times \delta_{l}+\sum_{c \in X_{d}} m_{d, c} L(c)\right) \rtimes\left(\gamma_{1} \times \ldots \times \gamma_{m} \rtimes \tau+\sum_{s \in Y_{t}} n_{t, s} L(s)\right) \\
=\delta_{1} \times \ldots \times \delta_{l} \times \gamma_{1} \times \ldots \times \gamma_{m} \rtimes \tau+\sum_{s \in Y_{t}} n_{t, s} \delta_{1} \times \ldots \times \delta_{l} \rtimes L(s) \\
+\sum_{c \in X_{d}} m_{d, c} L(c) \times \gamma_{1} \times \ldots \times \gamma_{m} \rtimes \tau+\sum_{c \in X_{d}} \sum_{s \in Y_{t}} m_{d, c} n_{t, s} L(c) \rtimes L(s)=
\end{gathered}
$$

$$
\begin{gathered}
\sum_{\tau^{\prime} \in T_{d, \tau}} k_{\tau^{\prime}} \delta_{1}^{\uparrow} \times \ldots \times \delta_{n}^{\uparrow} \times \gamma_{1} \times \ldots \times \gamma_{m} \rtimes \tau^{\prime} \\
\quad+\sum_{s \in Y_{t}} n_{t, s} \delta_{1} \times \ldots \times \delta_{l} \rtimes L(s) \\
+\sum_{c \in X_{d}} m_{d, c} L(c) \times \gamma_{1} \times \ldots \times \gamma_{m} \rtimes \tau
\end{gathered}
$$




$$
+\sum_{c \in X_{d}} \sum_{s \in Y_{t}} m_{d, c} n_{t, s} L(c) \rtimes L(s) .
$$

Now the representations in (4-7) show up as subquotients of representations in (4-9).

If $F$ is of characteristic 0 , then all $k_{\tau^{\prime}}$ are one (see [G] for split groups, the case of non-split groups goes as in $[\mathrm{H}]$ ). Now the properties of the Langlands classification imply that the representations in (4-7) show up as subquotients of representations in (4-9) with multiplicity one.

Therefore, for the proof of the proposition, it is enough to show that representations from (4-7) does not show up as subquotients of representations in the lines (4-10), (4-11) and (4-12). It is obvious that each irreducible subquotient of each representation in the line (4-12) is already a subquotient of some representation in the line (4-10) or (4-12) (actually, of the both). Therefore, for the proof of the proposition it is enough to show that representations from (4-7) does not show up as subquotients of the representations in the lines (4-10) and (4-11).

Observe that for each irreducible representation $\pi$ from (4-7), $\mathbf{e}(\pi)$ does not depend on $\tau^{\prime} \in T_{d, \tau}$. We shall show bellow that for each irreducible subquotient $\sigma$ of (4-10) or (4-11), $\mathbf{e}(\sigma)$ is strictly smaller that $\mathbf{e}(\pi)$, where $\pi$ is from (4-7). This will prove the proposition. Observe that we know

$$
e(\sigma) \leq e(\pi)
$$

by (4-6).

Suppose that some irreducible representation $\pi$ is a subquotient of some term in (4-11). Then it is a subquotient of some

$$
\left(\delta_{1} \times \ldots \times \delta_{i, j}^{\bigcap} \times \ldots \times \delta_{i, j}^{\sqcup} \times \ldots \times \delta_{l}\right) \times \gamma_{1} \times \ldots \times \gamma_{m} \rtimes \tau
$$

(see (4-2)). Observe $i \leq n$ since the tempered parabolic induction for general linear groups is irreducible.

Consider first the case $j \leq n$. Then the relation (4-1) and the definition of $\mathbf{e}(\pi)$ and $\mathbf{e}(\sigma)$ imply that $\mathbf{e}(\pi)<\mathbf{e}(\sigma)$ for $\sigma$ from (4-7).

Consider now $n<j$. Then $\pi$ is a subquotient of

$$
\left(\delta_{1} \times \ldots \times \delta_{i, j}^{\cap} \times \ldots \times \delta_{n} \times \ldots \times \delta_{i, j}^{\cup} \times \ldots \times \delta_{l}\right) \times \gamma_{1} \times \ldots \times \gamma_{m} \rtimes \tau .
$$

Write

$$
\left(\delta_{1}^{\uparrow}, \ldots, \delta_{n}^{\uparrow}, \gamma_{1}, \ldots, \gamma_{m} ; \tau^{\prime}\right)=\left(\beta_{1}, \ldots, \beta_{n+m} ; \tau^{\prime}\right)
$$

such that the right hand side is in a standard order (here $\tau^{\prime}$ is any element from $T_{d, \tau}$ ). Then $\delta_{i}^{\uparrow}=\beta_{k}$ for some $k$.

Let $\tau^{\prime \prime}$ be an irreducible subrepresentation of

$$
\delta_{n+1} \times \ldots \times \delta_{j-1} \times \delta_{j+1} \times \ldots \times \delta_{l} \rtimes \tau .
$$


If $e\left(\beta_{k}\right)>e\left(\beta_{k+1}\right)$ or $k=n+m$, writing $\left(\delta_{1}^{\uparrow}, \ldots,\left(\delta_{i, j}^{\bigcap}\right)^{\uparrow}, \ldots, \delta_{n}^{\uparrow},\left(\delta_{i, j}^{\sqcup}\right)^{\uparrow}, \gamma_{1}, \ldots, \gamma_{m} ; \tau^{\prime \prime}\right)$ in a standard order, relation (4-1) will imply directly

$$
\begin{aligned}
\mathbf{e}(\pi) \leq \mathbf{e}\left(\delta_{1}^{\uparrow}, \ldots,\left(\delta_{i, j}^{\cap}\right)^{\uparrow}, \ldots, \delta_{n}^{\uparrow},\left(\delta_{i, j}^{\cup}\right)^{\uparrow}, \gamma_{1}, \ldots, \gamma_{m} ; \tau^{\prime \prime}\right) \\
\quad<\mathbf{e}\left(\delta_{1}^{\uparrow}, \ldots, \delta_{n}^{\uparrow}, \gamma_{1}, \ldots, \gamma_{m} ; \tau^{\prime}\right)=\mathbf{e}\left(\beta_{1}, \ldots, \beta_{n+m} ; \tau^{\prime}\right)=\mathbf{e}(\sigma)
\end{aligned}
$$

for any $\sigma$ from (4-7).

If $e\left(b_{k}\right)=e\left(b_{k+1}\right)$, then look at all $\ell \geq k$ such that $e\left(\beta_{\ell}\right)=e\left(\beta_{k}\right)$. Let $\ell_{0}$ be maximal such index.

Write $\left(\delta_{1}^{\uparrow}, \ldots,\left(\delta_{i, j}^{\cap}\right)^{\uparrow}, \ldots, \delta_{n}^{\uparrow},\left(\delta_{i, j}^{\cup}\right)^{\uparrow}, \gamma_{1}, \ldots, \gamma_{m} ; \tau^{\prime \prime}\right)$ in a standard order, where $\tau^{\prime \prime}$ is an irreducible subrepresentation of $\delta_{n+1} \times \ldots \times \delta_{j-1} \times \delta_{j+1} \times \ldots \times \delta_{l} \rtimes \tau$ as before. We shall consider how from $\mathbf{e}\left(\beta_{1}, \ldots, \beta_{n+m} ; \tau^{\prime}\right)$ one gets

$$
\mathbf{e}\left(\delta_{1}^{\uparrow}, \ldots,\left(\delta_{i, j}^{\cap}\right)^{\uparrow}, \ldots, \delta_{n}^{\uparrow},\left(\delta_{i, j}^{\cup}\right)^{\uparrow}, \gamma_{1}, \ldots, \gamma_{m} ; \tau^{\prime \prime}\right) .
$$

Note that $\mathbf{e}\left(\delta_{1}^{\uparrow}, \ldots,\left(\delta_{i, j}^{\bigcap}\right)^{\uparrow}, \ldots, \delta_{n}^{\uparrow},\left(\delta_{i, j}^{\cup}\right)^{\uparrow}, \gamma_{1}, \ldots, \gamma_{m} ; \tau^{\prime \prime}\right)$ will have the same $\beta_{h}$ 's for $h<k$. Further, one needs to remove from $\mathbf{e}\left(\beta_{1}, \ldots, \beta_{n+m} ; \tau^{\prime}\right)$ elements $e\left(\beta_{k}\right)$ corresponding to $\beta_{k}$ and shift elements $e\left(\beta_{\ell}\right)$ corresponding to $\beta_{\ell}, k+1 \leq \ell \leq \ell_{0}$, to the left.

After $e\left(\beta_{\ell_{0}}\right)$ will come either $e\left(\beta_{\ell_{0}+1}\right)$ or $e\left(\left(\delta_{i, j}^{\cup}\right)^{\uparrow}\right)$ or $e\left(\left(\delta_{i, j}^{\uparrow}\right)^{\uparrow}\right)$. Now $e\left(\beta_{\ell_{0}+1}\right)<e\left(\beta_{\ell_{0}}\right)$ and (4-1) imply again (4-15), what we needed to prove.

To complete the proof, it remains to consider $\pi$ which is an irreducible subquotient of (4-10). Then it is a subquotient of some

$$
\delta_{1} \times \ldots \times \delta_{l} \rtimes L(s),
$$

where $L(s)$ is a subquotient of a representation which can have following two types.

The first type is is a representation for which there exist $1 \leq i<j \leq m$ and $\varepsilon_{i} \in\{ \pm 1\}$ such that $\left(\gamma_{i}^{\prime}\right)^{\varepsilon_{i}} \times \gamma_{j}^{\prime}$ reduces and that $L(s)$ is subquotient of

$$
\gamma_{1} \times \ldots \times\left(\gamma_{i, j, \varepsilon_{i}}\right)^{\cap} \times \ldots \times\left(\gamma_{i, j, \varepsilon_{i}}\right)^{\cup} \times \ldots \times \gamma_{l} \rtimes \tau .
$$

Again write $\left(\delta_{1}^{\uparrow}, \ldots, \delta_{n}^{\uparrow}, \gamma_{1}, \ldots, \gamma_{m} ; \tau^{\prime}\right)=\left(\beta_{1}, \ldots, \beta_{n+m} ; \tau^{\prime}\right)$ in a standard order (similarly as in (4-14)). Observe

$$
\left|e\left(\left(\gamma_{i, j, \varepsilon_{i}}\right)^{\cap}\right)\right|,\left|e\left(\left(\gamma_{i, j, \varepsilon_{i}}\right)^{\cup}\right)\right|<\max \left\{e\left(\gamma_{i}\right), e\left(\gamma_{j}\right)\right\} .
$$

Now from (4-1) and the above relation we prove (4-15) in the same way as in the previous case.

The remaining type of the representation is one for which there exists $i$ such that $\delta_{i}^{\prime} \rtimes \tau$ reduces, and after writing $\gamma_{i} \rtimes \tau=L\left(\gamma_{i} ; \tau\right)+K\left(\gamma_{i} ; \tau\right)$ in the Grothendieck group, then $L(s)$ is subquotient of

$$
\gamma_{1} \times \ldots \times \gamma_{i-1} \times \gamma_{i+1} \times \ldots \times \gamma_{m} \rtimes K\left(\gamma_{i} ; \tau\right) .
$$


Therefore, there exists a subquotient $\pi^{\prime}$ of $K\left(\gamma_{i} ; \tau\right)$ such that $L(s)$ is a subquotient of $\gamma_{1} \times \ldots \times \gamma_{i-1} \times \gamma_{i+1} \times \ldots \times \gamma_{m} \rtimes \pi^{\prime}$. We know

$$
\pi^{\prime}=L(r)
$$

for some $r \in M\left(D_{+}\right) \times T(S)$. Write

$$
r=\left(\mu_{1}, \ldots, \mu_{a} ; \tau^{\prime \prime}\right)
$$

in a standard order. Further,

$$
\mathbf{e}\left(\pi^{\prime}\right)=\mathbf{e}\left(\mu_{1}, \ldots, \mu_{a} ; \tau^{\prime \prime}\right)=\left(x_{1}, \ldots, x_{b}\right)<\mathbf{e}\left(\gamma_{i}, \tau\right)=(\underbrace{e\left(\gamma_{i}\right), \ldots, e\left(\gamma_{i}\right)}_{u \text { times }}, 0, \ldots, 0)
$$

where $\gamma_{i}$ is a representation of $G L(u, F)$. This implies

$$
\begin{array}{ll}
x_{1} \geq \cdots \geq x_{b} \geq 0 ; & x_{1} \leq e\left(\gamma_{i}\right) \\
x_{1}+\cdots+x_{u}<u e\left(\gamma_{i}\right) ; & x_{1}+\cdots+x_{b} \leq u e\left(\gamma_{i}\right) .
\end{array}
$$

The third relation implies that the number $v$ of indices $j$ such that $x_{j}=e\left(\gamma_{i}\right)$ satisfies

$$
v<u
$$

We can write now in a the standard order

$$
\left(\delta_{1}, \ldots, \delta_{n}, \gamma_{1}, \ldots, \gamma_{i-1}, \gamma_{i+1}, \ldots, \gamma_{m}, \mu_{1}, \ldots, \mu_{a}, \tau^{\prime \prime \prime}\right)
$$

where $\tau^{\prime \prime \prime}$ is an irreducible subrepresentation of $\delta_{n+1} \times \ldots \times \delta_{l} \rtimes \tau^{\prime \prime}$. Now from (4-19) one sees similarly as above that $\mathbf{e}(\pi)<\mathbf{e}(\sigma)$ for $\sigma$ from (4-7). The proof is now complete

4.3. Remarks. (i) The above proposition holds also in the archimedean case, in unchanged form. The proof there goes the same way as in the non-archimedean case, except that we need to make small modifications regarding composition series of representations $\delta_{1} \times \delta_{2}, \delta_{i} \in D$.

(ii) The above proposition holds also for hermitian quaternionic groups in the case of characteristic 0 .

(iii) The second claim of the above proposition holds also in positive characteristic. This follows from the work of of J.-L. Waldspurger and V. Heiermann, but for this we do not have a written reference (the result that one needs is that standard intertwining operators span the intertwining algebra of the representation induced by an irreducible square integrable one).

Easiest way of constructing irreducible unitarizable representations of reductive groups is parabolic induction starting with irreducible unitarizable representations of proper Levi subgroups. For this, we need to know when such representations reduce, and what are irreducible constituents. Natural place to start construction for classical groups $S_{m}$ 's are 
representations $\pi \rtimes \tau$ where $\pi$ is an irreducible unitarizable representation of a general linear group (such representations are classified) and $\tau$ is an irreducible tempered representation of a classical group $S_{m}$ (we would like to start with more general unitarizable representations $\tau$ 's, but we do not know many much more general irreducible unitarizable representations of classical groups).

For a unitarizable $\delta \in D$ and a positive integer $n$ denote

$$
u(\delta, n)=L\left(\nu^{(n-1) / 2} \delta \times \nu^{(n-1) / 2-1} \delta \times \ldots \times \nu^{-(n-1) / 2} \delta\right) .
$$

Then $u(\delta, n)$ is a unitarizable representation of a general linear group. These representations play a crucial role in the description of the unitary duals of general linear groups (see [T1]). Each irreducible unitarizable representation of a general group is induced from a tensor product of such representations or complementary series starting with such representations.

Let us return to the classical groups and representations $\pi \rtimes \tau$ where $\pi$ is an irreducible unitarizable representation of a general linear group and $\tau$ is an irreducible tempered representation of a classical groups $S_{m}$. We can handle reducibility questions of representations $\pi \rtimes \tau$ when $\pi$ 's are complementary series pretty well using Lemma 3.4 and Proposition 2.2. Therefore, we shall concentrate now to the problem of reducibility of representations $u(\delta, n) \rtimes \tau$.

The above proposition enables us to get easily a partial information in that direction. Let us note that we have a description of reducibility points of representations $\delta^{\prime} \rtimes \tau$ for $\delta \in D$ and $\tau$ irreducible square integrable representation, and also if $\tau$ is an irreducible tempered representation (see [Mu1], [Mu2]).

4.4. Corollary. Suppose that $\tau$ is an irreducible representation of a classical group $S_{m}$, $\delta \in D$ is unitarizable and that $n$ is non-negative integer.

(i) Let $\delta_{1}, \ldots, \delta_{k} \in D$ be unitarizable and not self dual (i.e. $\delta_{i} \neq \tilde{\delta}_{i}, i=1, \ldots, k$ ). If $n_{1}, \ldots, n_{k}$ are positive integers and $\tau$ is weakly real or tempered, then

$$
u\left(\delta_{1}, n_{1}\right) \times \ldots \times u\left(\delta_{k}, n_{k}\right) \rtimes \tau
$$

is irreducible.

(ii) Suppose that $\tau$ is tempered and that $\delta \rtimes \tau$ reduces. Then

$$
u(\delta, 2 n+1) \rtimes \tau
$$

reduces.

(iii) Suppose that $\tau$ is tempered. Then $u(\delta, 2) \rtimes \tau$ reduces if and only if $\nu^{1 / 2} \delta \rtimes \tau$ reduces. (iv) Suppose that $\tau$ is tempered and that $\delta \rtimes \tau$ and $\nu \delta \rtimes \tau$ are irreducible. Then $u(\delta, 3) \rtimes \tau$ is irreducible.

Proof. (i) We get easily (i) if $\tau$ is weakly real from Proposition 2.2 (see the proof of Theorem 3.2). If $\tau$ is tempered, then $\tau \cong \delta_{1}^{\prime} \times \ldots \times \delta_{k^{\prime}}^{\prime} \rtimes \tau^{\prime}$, where $\delta_{i}^{\prime} \in D$ are unitary and not self dual, and $\tau^{\prime}$ is weakly real. Now the first part of the proof implies irreducibility.

(ii) Proposition 4.2 implies (ii) directly. 
(iii) Note that $L\left(\nu^{1 / 2} \delta, \tau \nu^{1 / 2} \delta\right.$; $\left.\tau\right)$ is always a subquotient of $u(\delta, 2) \rtimes \tau$ by the above proposition. Suppose that $\nu^{1 / 2} \delta \rtimes \tau$ reduces. Then there exists $t \in M\left(D_{+}\right) \times T(S), t \neq$ $\left(\nu^{1 / 2} \delta, \tau\right)$ such that $L(t)$ is a subquotient of $\nu^{1 / 2} \delta \rtimes \tau$. Now $L\left(\left(\nu^{1 / 2} \delta\right)+t\right)$ is a subquotient of $\nu^{1 / 2} \delta \times \nu^{1 / 2} \delta \rtimes \tau$ by the above proposition. This is a non-tempered representation. From the representation theory of general linear groups we know that $\nu^{1 / 2} \delta \times \nu^{1 / 2} \delta \rtimes \tau$ consists of $u(\delta, 2) \rtimes \tau$ and a tempered part. Therefore, $L\left(\left(\nu^{1 / 2} \delta\right)+t\right)$ is a subquotient of $u(\delta, 2) \rtimes \tau$. Since $\left(\nu^{1 / 2} \delta\right)+t$ is different from $\left(\nu^{1 / 2} \delta, \tau \nu^{1 / 2} \delta ; \tau\right)$, we get that $u(\delta, 2) \rtimes \tau$ is reducible.

Suppose that $\nu^{1 / 2} \delta \rtimes \tau$ is irreducible. Now we have surjective intertwining

$$
\nu^{1 / 2} \delta \times \nu^{1 / 2} \delta \rtimes \tau \cong \nu^{1 / 2} \delta \times \nu^{-1 / 2} \delta \rtimes \tau \rightarrow u(\delta, 2) \rtimes \tau .
$$

Since the first representation has unique irreducible quotient, $u(\delta, 2) \rtimes \tau$ must also have unique irreducible quotient. Since the last representation is unitarizable, it must be irreducible.

(iv) Using Zelevinsky involution, the fact that it carries irreducible representations to the irreducible ones, and Gelfand-Kazhdan derivatives, we get easily that $L(\nu \delta, \delta) \times \delta$ is irreducible. Now we have surjective intertwinings

$\nu \delta \times \nu \delta \times \delta \rtimes \tau \rightarrow \nu \delta \times L(\nu \delta, \delta) \rtimes \tau \cong L(\nu \delta, \delta) \times \nu \delta \rtimes \tau \cong L(\nu \delta, \delta) \times \nu^{-1} \delta \rtimes \tau \rightarrow u(\delta, 3)$

This implies irreducibility (use the same argument as in the proof of (iii)).

4.5. Remarks. (i) After the above corollary, it is natural to ask: is $u(\delta, n) \rtimes \pi$ irreducible if $\delta^{\prime} \rtimes \pi$ are irreducible for all $\delta^{\prime} \in\left\{\nu^{(n-1) / 2} \delta, \nu^{(n-1) / 2-1} \delta, \ldots, \nu^{-(n-1) / 2} \delta\right\}$.

(ii) After understanding reducibilities $u(\delta, n) \rtimes \tau$, next question would be to understand representations $u\left(\delta_{1}, n_{1}\right) \times \ldots \times u\left(\delta_{k}, n_{k}\right) \rtimes \tau$. Note that the theory of $R$ groups takes care of the case $n_{1}=\cdots=n_{k}=1$. In this case we get a representation of length $2^{l}$ for some $l$ (which we can describe in term of relative rank one reducibilities). The general situation will be different. We shall give an example.

For a connected reductive group $G, 1_{G}\left(\operatorname{resp} . \mathrm{St}_{G}\right)$ will denote the trivial one dimensional (resp. Steinberg) representation of $G$. Now we shall consider split odd-orthogonal groups. It is not hard to see that the representation $\mathrm{St}_{G L(2, F)} \rtimes \mathrm{St}_{S O(3, F)}$ is irreducible, and that $1_{G L(2, F)} \rtimes \mathrm{St}_{S O(3, F)}$ reduces into two irreducible pieces (clearly, $1_{G L(2, F)} \times \mathrm{St}_{G L(2, F)}$ is irreducible). D. Ban and C. Jantzen proved in [BnJ] that

$$
1_{G L(2, F)} \times \mathrm{St}_{G L(2, F)} \rtimes \mathrm{St}_{S O(3, F)}=u\left(1_{G L(1, F)}, 2\right) \times u\left(\mathrm{St}_{G L(2, F)}, 1\right) \rtimes \mathrm{St}_{S O(3, F)}
$$

reduce into three irreducible pieces.

(iii) If we fix a self dual $\delta$, we shall have often $u(\delta, n) \rtimes \tau$ reducible. For example, at least one of the representations $u(\delta, 2 n+1) \rtimes 1_{S O(1, F)}$ or $u(\delta, 2 n+1) \rtimes 1_{S p(0, F)}$ is always reducible (this follows from the duality established by F. Shahidi in [Sh2]).

\section{A Simple CASE OF THE REDUCiBility PROBlem}

Let us first recall of a classification of irreducible essentially square integrable representations of general linear groups (see [Z]). For $\rho \in \mathcal{C}$ and a positive integer $n$, the set $\Delta=$ 
$\left[\rho, \nu^{n} \rho\right]=\left\{\rho, \nu \rho, \ldots, \nu^{n} \rho\right\}$ is called a segment in $\mathcal{C}$. The representation $\nu^{n} \rho \times \nu^{n-1} \rho \times \ldots \times \rho$ contains the unique irreducible subrepresentation, which will be denoted by $\delta(\Delta)$. It is essentially square integrable. Segments in $\mathcal{C}$ parameterize $D(\Delta \mapsto \delta(\Delta)$ is a bijection).

We have seen that for understanding the unitarizability is very useful to understand the reducibility of parabolic induction. One of the first steps in understanding reducibility for classical $p$-adic groups, is to understand reducibility of

$$
\delta \rtimes \pi
$$

where $\delta$ and $\pi$ are irreducible essentially square integrable representations ( $\delta$ of a general linear group and $\pi$ of a classical group). This question is in general hard and complicated (see [Mu1], [Mu2]). If $\pi$ is cuspidal, the problem of reducibility of (5-1) has a simple answer (reduction): if (HI) holds (and $\pi$ is cuspidal), then

$$
\delta(\Delta) \rtimes \pi \text { is reducible if and only if } \rho \rtimes \pi \text { reduces for some } \rho \in \Delta \text {. }
$$

The opposite case to $\pi$ cuspidal is the case of $\delta$ cuspidal. We shall describe briefly the reducibility in this case.

Let $\rho$ be an irreducible unitarizable cuspidal representation of $G L(p, F)$ and let $\pi$ be an irreducible square integrable representation of $S_{q}$. If $\rho$ is not self dual, then $\nu^{\alpha} \rho \rtimes \pi$ is irreducible for all $\alpha \in \mathbb{R}$. Therefore, we shall assume in the sequel that $\rho$ is self dual.

In the sequel we shall assume that assumption (BA) from $[\mathrm{M} \mathrm{T}]$ holds.

For a positive integer $a$ denote $\delta(\rho, a)=\delta\left(\left[\nu^{-(a-1) / 2} \rho, \nu^{(a-1) / 2} \rho\right]\right)$. In [Mœ] is defined parity of $\rho$ with respect to $\pi$ (see [MœT] also). Parity can be characterized in the following way: it is even (resp. odd) if $\delta(\rho, a) \rtimes \pi$ is irreducible for all odd (resp. even) positive integers $a$. Further, $\operatorname{Jord}(\pi)$ is defined as the set of all pairs $(\rho, a)$ where $a$ is a positive integer of the same parity as the parity of $\rho$ with respect to $\pi$, such that $\delta(\rho, a) \rtimes \pi$ is irreducible (see [Mœ], and also [MœT]). Further, C. Mœglin has introduced partially defined function $\varepsilon_{\pi}$. Then (admissible) triples $\left(\operatorname{Jord}(\pi), \varepsilon_{\pi}, \pi_{\text {cusp }}\right)$ parameterize irreducible square integrable representations of groups $S_{n}$ 's (see [MœT] for details). In the following proposition we collect the facts about reducibility of representations (5-1) when $\delta$ is cuspidal.

5.1. Proposition. Let $\rho \in \mathcal{C}$ be self dual, and let $\pi$ be an irreducible square integrable representation of a group $S_{q}$. Suppose that (BA) from [MœT] holds. Denote $\operatorname{Jord}_{\rho}(\pi)=$ $\left\{k ;(\rho, k) \in \operatorname{Jord}_{\rho}(\pi)\right\}$. Let a be a positive integer. Then:

(i) For $\alpha \in \mathbb{R}, \nu^{\alpha} \rho \rtimes \pi$ reduces if and only if $\nu^{-\alpha} \rho \rtimes \pi$ reduces.

(ii) If $\alpha \in \mathbb{R} \backslash(1 / 2) \mathbb{Z}$, then $\nu^{\alpha} \rho \rtimes \pi$ is irreducible.

(iii) $\rho \rtimes \pi$ reduces if and only if $\rho$ has odd parity with respect to $\pi$ and $1 \notin \operatorname{Jord}_{\rho}(\pi)$.

(iv) If $a \notin \operatorname{Jord}_{\rho}(\pi)$, then $\nu^{(a+1) / 2} \rho \rtimes \pi$ is irreducible.

(v) If $a \in \operatorname{Jord}_{\rho}(\pi)$ and $a+2 \notin \operatorname{Jord}_{\rho}(\pi)$, then $\nu^{(a+1) / 2} \rho \rtimes \pi$ is reducible.

(vi) Suppose that $a$ and $a+2$ are in $\operatorname{Jord}_{\rho}(\pi)$. Then $\nu^{(a+1) / 2} \rho \rtimes \pi$ is reducible if and only if $\varepsilon_{\rho}(a)=\varepsilon_{\rho}(a+2)$.

(vii) $\nu^{1 / 2} \rho \rtimes \pi$ is reducible if and only if $2 \notin \operatorname{Jord}_{\rho}(\pi)$ or $2 \in \operatorname{Jord}_{\rho}(\pi)$ and $\varepsilon_{\rho}(2)=1$. In other words, $\nu^{1 / 2} \rho \rtimes \pi$ is irreducible if and only if $2 \in \operatorname{Jord}_{\rho}(\pi)$ and $\varepsilon_{\rho}(2)=-1$. 
The first claim is a consequence of the structure of $R$ module $R(S)$ (see the first section). The second claim is well known (it follows also from Lemma 3.4). The third claim is is just a definition of $\operatorname{Jord}(\pi)$. The fourth claim is (iv) Lemma 5.4.1 of [Mœ], while the fifth claim is Lemma 5.3 of [MœT]. In the remark after the proof of the Lemma 5.3 in [MœT] is a sketch of proof of sixth claim. That proof is based on intertwining operators. We have also a proof based on Jacquet module techniques of [T7]. The seventh claim also follows applying these techniques.

G. Muić has described completely reducibility points of representations (5-1) in [Mu1] (in [Mu2] he settles the case of more general case of standard modules). The above proposition is a very special (starting) case of his results. The above proposition is elementary in comparison with his results. The same comment is valid for the following simple lemma, which is sometimes useful. It can be proved in a rather simple way.

5.2. Lemma. Assume that (BA) holds. Let $\Delta$ be a segment in irreducible cuspidal representations of general linear groups and let $\pi$ be an irreducible square integrable representation of a group $S_{q}$. Suppose that $\rho \rtimes \pi$ is irreducible for all $\rho \in \Delta$. Then

$$
\delta(\Delta) \rtimes \pi
$$

is irreducible.

\section{REFERENCES}

[BnJ] Ban, D. and Jantzen, C., R-groups in the non-tempered case, preprint (2005).

[Bn] Bernstein J., P-invariant distributions on $G L(N)$ and the classification of unitary representations of $G L(N)$ (non-archimedean case), Lie Group Representations II, Proceedings, University of Maryland 1982-83, Lecture Notes in Math. 1041, Springer-Verlag, Berlin, 1984, pp. 50-102.

[BrMo1] D. Barbasch, A. Moy, Reduction to real infinitensimal character in affine Hecke algebras, Journal of A.M.S. 6 (1993), 611-635.

[BrMo2] D. Barbasch, A. Moy, Unitary spherical spectrum for p-adic classical groups, Acta Appl. Math. 44 (1996), no. 1-2, 3-37.

[C] Casselman, W., Introduction to the theory of admissible representations of p-adic reductive groups, preprint.

[H] Hanzer, M., R-groups for Hermitian Quaternionic Groups, Glasnik Matematicki 39(59) (2004), no. $1,31-48$.

[G] Goldberg, D., Reducibility of induced representations for $S p(2 n)$ and $S O(n)$, Amer. J. Math. 116 (1994), no. 5, 1101-1151.

[J] Jantzen, C., On supports of induced representations for symplectic and odd-orthogonal groups, Amer J. Math. 119 (1997), 1213-1262.

[LMuT] Lapid, E., Muić G. and Tadić, M., On the generic unitary dual of quasisplit classical groups, Int. Math. Res. Not. 26 (2004), 1335-1354.

[Mœ] Møglin, C., Sur la classification des séries discrètes des groupes classiques p-adiques: paramètres de Langlands et exhaustivité., J. Eur. Math. Soc. 4 (2002), no. 2, 143-200.

[MœT] Møglin, C. and Tadić, M., Construction of discrete series for classical p-adic groups, J. Amer. Math. Soc. 15 (2002), 715-786.

[MœVW] Møglin, C.; Vignéras, M.-F. and Waldspurger, J.-L., Correspondances de Howe sur un corps p-adique, Lecture Notes in Math. 1291, Springer-Verlag, Berlin, 1987.

[Mu1] Muic, G., Reducibility of Generalized Principal Series, Canad. J. Math. 57 (2005), 616-647.

[Mu2] _ Reducibility of Standard Representations, Pacific. J. Math. 222 (2005), 133-168 (to appear). 
[Mu3] On the Non-Unitary Unramified Dual for Classical p-adic Groups, Trans. Amer. Math. Soc. 358 (2006), 4653-4687.

[Mu4] On Certain Classes of Unitary Representations for Split Classical Groups, Canadian J. Math. (to appear).

[Sh1] Shahidi, F., A proof of Langlands conjecture on Plancherel measures; complementary series for p-adic groups, Ann. of Math. 132 (1990), 273-330.

[Sh2] Shahidi, F., Twisted endoscopy and reducibility of induced representations for p-adic groups, Duke Math. J. 66 (1992), 1-41.

[T1] Tadić, M., Classification of unitary representations in irreducible representations of general linear group (non-archimedean case), Ann. Sci. École Norm. Sup 19 (1986), 335-382.

[T2] Unitary representations of general linear group over real and complex field, preprint MPI/SFB 85-22 Bonn (1985).

[T3] Geometry of dual spaces of reductive groups (non-archimedean case), J. Analyse Math. 51 (1988), 139-181.

[T4] Structure arising from induction and Jacquet modules of representations of classical p-adic groups, Journal of Algebra 177 (1995), no. 1, 1-33.

[T5] - Representations of p-adic symplectic groups, Compositio Math. 90 (1994), 123-181.

[T6] _ An external approach to unitary representations, Bulletin Amer. Math. Soc. 28 (1993), no. 2, 215-252.

[T7] _ On reducibility of parabolic induction, Israel J. Math. 107 (1998), 29-91.

[T8] - On regular square integrable representations of p-adic groups, Amer. J. Math. 120 (1998), no. 1, 159-210.

[T9] - On classification of some classes of irreducible representations of classical groups, Representations of real and $p$-adic groups, Singapore University Press and World Scientific, Singapore, 2004, pp. 95-162.

[Z] Zelevinsky, A. V.:, Induced representations of reductive p-adic groups II. On irreducible representations of $G L(n)$, Ann. Sci. École Norm. Sup. 13 (1980), 165-210. 